\title{
Mise en évidence d'un Sénonien gypseux sous la série phosphatée du bassin des Ouled Abdoun: Un nouveau point de départ pour l'origine des zones dérangées dans les mines à ciel ouvert de Khouribga, Maroc
}

\author{
Description of a gypseous Senonian under the phosphatic series of the \\ Ouled Abdoun basin: A new starting point for the origin of the disturbed \\ areas in the open sky mines of Khouribga, Morocco
}

\author{
N. El Assel ${ }^{1}$, A. Kchikach ${ }^{1}$, C. Durlet ${ }^{2}$, N. AlFedy ${ }^{2}$, K. El Hariri ${ }^{1}$, M. Charroud ${ }^{3}$, M. Jaffal ${ }^{1}$, \\ E. Jourani ${ }^{4}$, M. Amaghzaz ${ }^{4}$
}

\begin{abstract}
RÉSUMÉ
Dans le bassin sédimentaire des Ouled Abdoun (Maroc), la série phosphatée est formée d'une intercalation régulière de niveaux phosphatés et marno-calcaires sur environ $50 \mathrm{~m}$ de puissance. Localement, cette régularité est perturbée par la présence fréquente de structures communément appelés " dérangements ». II s'agit de masses non stratifiées, stériles, qui perturbent et alourdissent les travaux d'exploitation des couches phosphatées. Les études géophysiques expérimentales, réalisées dans les zones dérangées de la série phosphatée, ont démontré la possibilité de les cartographier sous couvertures à l'échelle décamétrique. Leur généralisation sur toute la superficie du bassin des Ouled Abdoun, nécessite une compréhension du phénomène à l'origine de ces structures.

Notre étude concerne l'analyse sédimentologique et diagénétique des zones dérangées et de leur voisinage immédiat. Elle a permis d'identifier pour la première fois l'existence d'une série évaporitique intensément karstifiée au sommet du Sénonien, sous la série phosphatée. Les karsts sénoniens sont incontestablement à l'origine des dérangements ou fontis que l'on défini comme étant des phénomènes de collapses à l'aplomb de cavités souterraines. Ces fontis ont une structuration polyphasée et une genèse au dépend de plusieurs processus (fracturation, infiltration, dissolution, effondrement). Leur mise en place a débuté dès la fin du Sénonien et s'est poursuivie jusqu'au quaternaire.
\end{abstract}

Mots-clefs: série phosphatée, zone dérangée, Sénonien, gypse, karst, Khouribga, Maroc.

\begin{abstract}
In the Ouled Abdoun sedimentary basin (Morocco), the phosphatic series is composed of regular interbedded phosphatic and marly limestone layers. Exploitation of the phosphate in some deposits in this basin collides frequently with problems bound to the existence, in the phosphatic series, of disturbed areas (sterile bodies) qualified as derangements by the mining engineers of the Office Cherifian Phosphate Group (OCP). Their presence in the phosphatic layers causes two kinds of problems: (1) since the whole phosphatic sequence is overlain by a Quaternary cover, we do not know their volume proportion in the phosphatic layers, and therefore the reserves estimations can be wrong (2) they are generally hard, so they complicate the phosphate extraction. Indeed, in an area containing sterile bodies, boring grid may always be tightened and boreholes filled with dynamite. The required borehole tools and use of explosives are time consuming and therefore increase drastically the cost of phosphate extraction. Their localisation would permit the mining engineers to get around them during the exploitation.
\end{abstract}

\footnotetext{
${ }^{1}$ Equipe de recherche E2G, Faculté des Sciences et Techniques, Université Cadi Ayyad, Marrakech, Maroc. Email: a.kchikach@uca.ma; kchikach@gmail.com

${ }^{2}$ Centre des Sciences de la Terre, Université de Bourgogne, Dijon, France.

${ }^{3}$ Université Sidi Mohamed Ben Abdellah, Faculté des Sciences et Techniques, Fès, Maroc

${ }^{4}$ Groupe Office Chérifien des Phosphates (OCP), Khouribga, Maroc
} 


\begin{abstract}
Several geophysical works have carried out in the achieved in Khouribga area to localize and delimit these sterile bodies. Electric resistitivy was established as a suitable geophysical parameter to map them, but the slowness and difficulty of data collection hinders the application of these geophysical methods to the whose phosphatic deposits area (about 25000 ha). Their application in all the Ouled Abdoun basin requires the comprehension of the origin of the sterile bodies, in order to specify their formation process and to predict consequently their spacial distribution in each phosphatic deposit.

Our study concerns to sedimentological and diagenetic analysis of disturbed aereas and their immediate vicinity. It made it possible to identify for the first time the existence of an evaporitic series intensely karstified at the top of the Senonian, under the phosphatic series. The senonian karsts are undoubtedly at the origin of the derangements, which are defined as the collapsing phenomena at the base of underground cavities.

These bodies have a polyphase structuring and a genesis that is the consequence of on several processes (fracturing, infiltration, dissolution, collapse). Their installation began from the end of Senonian, and continues up to the Quaternary.
\end{abstract}

Keywords: Phosphatic series, disturbed area, Senonian, gypsum, karst, Khouribga, Morocco.

\section{Introduction}

Le bassin des Ouled Abdoun, sur la Meseta marocaine (Fig. 1a), renferme les plus grands gisements de phosphate au monde. Il s'étend entre $6^{\circ}$ et $7^{\circ} 35^{\prime}$ de longitude ouest, et $32^{\circ} 15^{\prime}$ et $33^{\circ}$ de latitude nord. Son importance tient non seulement à sa vaste superficie mais aussi aux teneurs élevées en phosphates marins que l'on y rencontre (Boujo, 1976; Sheldon et al., 1989; Moutaouakil, 1990; Moutaouakil \& Giresse, 1993). Outre cet aspect économique, ce gisement est aussi très étudié d'un point de vue paléontologique, du fait de sa grande richesse en fossiles de vertébrés marins et terrestres d'âge maestrichtien à yprésien (Azmany et al., 1986; Cavin et al., 2000; Gheerbrant et al., 2003; Suberbiola et al., 2003; Bardet et al., 2004).

Les phosphates les plus purs, azoïques ou fossilifères (essentiellement des marnes phosphatées et des phospharénites marines), sont interstratifiés avec des bancs stériles sub-horizontaux constitués de calcaires à nodules et bancs discontinus de silex, de dolomites et de marnes. Cettee régularité du gisement stratiforme n'est toutefois pas systématique; dans les gisements en cours d'exploitation et dans plusieurs endroits du bassin des Ouled Abdoun, en particulier le secteur situé à l'Ouest et au Sud-Ouest de la ville de Oued Zem (Fig. 1a), la régularité des bancs phosphatés est fréquemment perturbée par des flexurations et des vastes corps stériles non stratifiés, qui interrompent ou déforment les couches phosphatées. Appelées zones dérangées ou tout simplement dérangements (Fig. 1b) par les géominiers du groupe Office Chérifien des Phosphates (OCP), ces corps sont à l'origine de deux types de problèmes: (i) Par leur aspect souvent dur et compact, ils perturbent la chaine d'exploitation et alourdissent le prix de revient d'extraction des phosphates. En effet, au cours d'une opération de forage, ou de «sautage » le long des tranchées d'exploitation, en présence d'un dérangement rhéologiquement plus résistant, les trous remplis d'explosifs doivent être plus serrés. Lors de l'extraction sélective du phosphate, les dérangements étant parfois indestructibles même après sautage, causent un vrai problème en ce qui concerne le libre déplacement des draglines dans les découvertes. (ii) Du fait qu'ils sont stériles et toujours masqués par une couverture quaternaire, ils ne permettent pas de réaliser un calcul précis des réserves et par conséquent une bonne étude de faisabilité pour chaque gisement.

La prospection et la délimitation des zones dérangées préoccupent les ingénieurs miniers et les géologues de l'OCP. Leur localisation et délimitation permettrait de les contouner lors de l'exploitation des couches phosphatées. Les études géophysiques expérimentales (Kchikach et al., 2002 \& Kchikach et al., 2006) réalisées sur les dérangements ont démontré la possibilité de les cartographier sous la couverture quaternaire. La généralisation de ces études sur l'ensemble des gisements des Ouled Abdoun nécessite la compréhension du processus à l'origine des dérangements pour orienter les travaux géophysiques de prospection vers les zones les plus susceptibles d'en contenir et aider par consequent à une meilleure exploitation des gisements phosphatés.

A ce jour, l'origine des dérangements est restée problématique et très peu étudiée. Des hypothèses peu étayées ont fait appel soit à des phénomènes 
d'érosion synsédimentaire qui donnent lieu à des paléochenaux (El Mountassir, 1977), soit à des processus diagénétiques et/ou pédologiques tardifs qui donnent naissance à des paléochenaux impliquant la dissolution de calcaires et de marnes interstratifiés dans la série phosphatée (Lucas et al., 1979; Boujo, 2002). Une autre hypothèse envisage l'effondrement de cavités karstiques situées au sein des calcaires du Turonien (El Felah, 1990) (Fig. 1c). Bien que nombreux et ayant un impact certain sur les ressources hydrologiques de la région (Hsissou et al., 1996; Ettazarini, 2004), les karsts turoniens ne semblent pas avoir une incidence directe sur la série phosphatée car ils forment des cavités mécaniquement stables (peu effondrées) et situés à plus de 150 mètres de profondeur au dessous de cette dernière.

Dans cet article, nous proposons et argumentons un autre processus pour expliquer la présence des derangements dans la série phosphatée. Nos observations mettent en cause des phénomènes karstiques polyphasés causés par des dissolutions affectant des couches de gypses situées immédiatement sous la série phosphatée et totalement inconnues jusqu'alors. A partir d'une étude sédimentaire et diagénétique du remplissage et des encaissants des dérangements, nous proposons: de décrire pour la première fois la série gypseuse du Sénonien le long d'une coupe de $30 \mathrm{~km}$ dans la région de KhouribgaOued Zem; de présenter les phénomènes de dissolution affectant cette série sénonienne; d'esquisser une chronologie des principales phases de karstification affectant les couches sus-jacentes au Sénonien; d'expliquer et de dater la présence des déformations et des corps stériles qui perturbent l'exploitation des phosphates et de discuter des possibilités d'orienter les travaux géophysiques de prospection des dérangements par l'étude du soubassement évaporitique de la série phosphatée.

\section{Contexte géologique}

Le gisement des Ouled Abdoun, aussi surnommé plateau des Ourdirha, constitue la partie septentrionale du bassin sédimentaire de l'Oum Rabia. Parmi les gisements phosphatés marocains, il est le plus anciennement connu (1917) et exploité (1921). Il comporte une série sédimentaire quasi tabulaire d'âge crétacé-supérieur à éocène (Michard, 1976 \& Zouhri et al., 2008) qui repose en discordance sur le socle hercynien de la partie occidentale de la Meseta

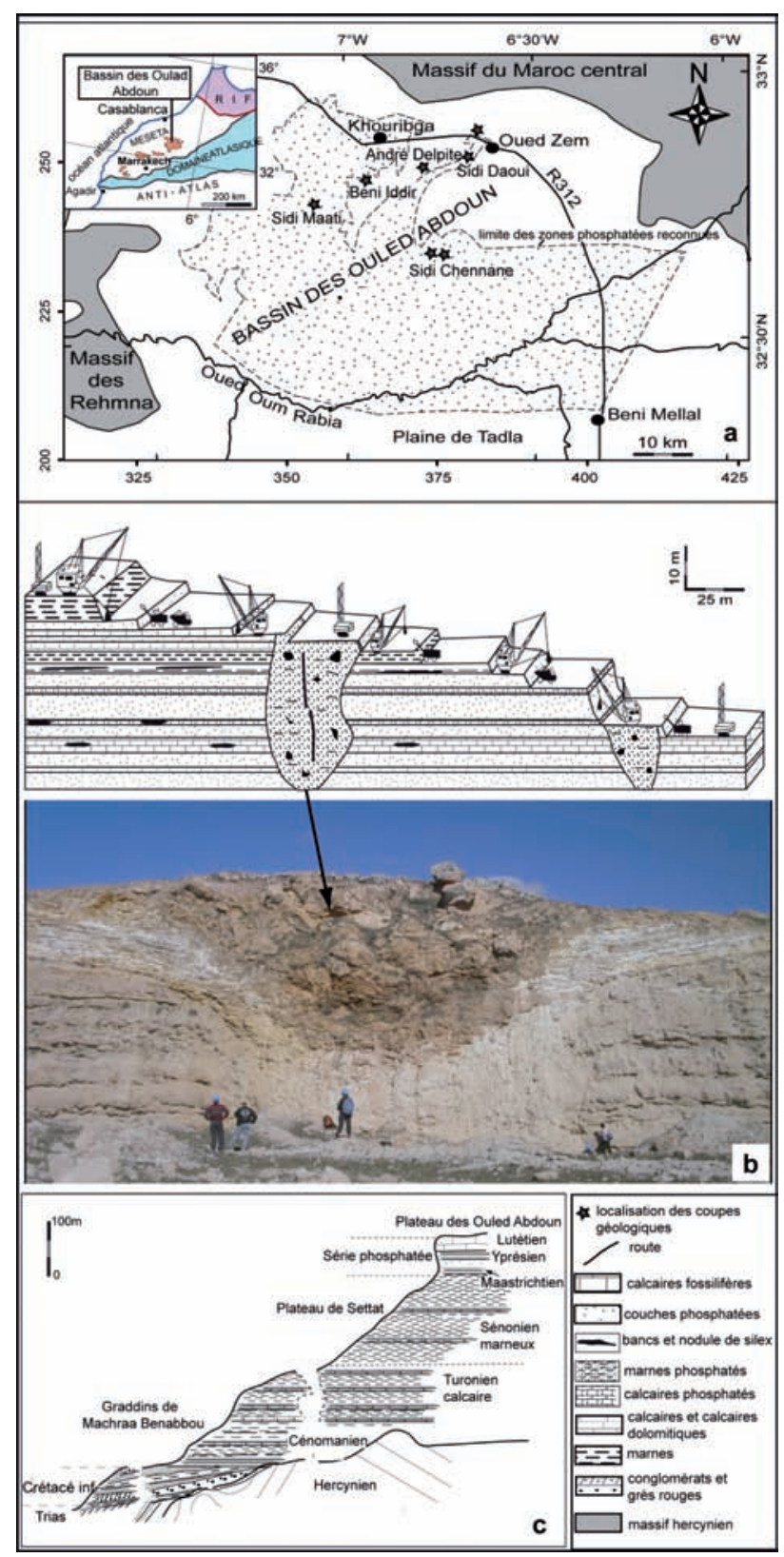

Fig. 1.-a. Localisation des zones étudiées dans le bassin des Ouled Abdoun. b. Schéma d'une tranchée d'exploitation montrant un dérangement. c. Place de la série phosphatée dans le contexte stratigraphique régionale (d'après Michard, 1976).

marocaine (Fig.1c). Peu épaisse, la série sédimentaire des Ouled Abdoun est circonscrite au nord et à l'ouest par les affleurements paléozoïques de la Meseta, et au sud-est par les escarpements jurassiques du Moyen et du Haut Atlas. Au sud, cette série est enfouie sous les dépôts continentaux du Miocène, Pliocène et Quaternaire de la plaine du Tadla. 
A proximité des villes de Khouribga et d'Oued Zem, cette série sédimentaire est traditionnellement décrite (Azmany, 1979) comme comportant cinq grands ensembles lithotologiques superposés, de bas en haut :

(i) une série cénomanienne complexe où alternent marnes et calcaires;

(ii) une série turonienne dominée par des dolomites et des calcaires massifs à rares intercalations marneuses, affectés par des phénomènes karstiques.

(iii) une série dite sénonienne, mais vraisemblablement d'âge coniacien à campanien, essentiellement marneuse avec quelques intercalations calcaires ;

(iv) une série d'âge campanien à yprésien qui comporte des bancs phosphatés et des inter-bancs stériles ;

(v) une série lutétienne principalement constituée de biocalcarénites marines lithostratigraphiquement regroupées au sein de la « dalle à Thersitées » (gastéropodes marins des genres Hemithersitea et Thersitea $s p$ ).

La série phosphatée proprement dite (Fig. 2) débute par des marnes phosphatées et des niveaux calcaires très riches en débris osseux, connus sous le nom de calcaires à bone-beds d'âge maastrichtien (Noubhani et al., 1995; Suberbiola et al., 2003). Cet étage marque le début d'une phosphatogenèse qui atteint son maximum dans les étages suivants. Au dessus vient une succession de niveaux de phosphates meubles grossiers et de calcaires phosphatés; le toit du Maastrichtien est formé de calcaires marneux et de marnes grises. Le Montien est représenté par des phosphates meubles surmontés par des calcaires à coprolithes et à nodules de silex, qui constituent un niveau repère pour l'exploitation minière. Ce dernier est surmonté par une alternance de bancs réguliers de calcaires marneux et phosphatés, de niveaux de phosphates meubles à grains grossiers, d'horizons continus de silex et, parfois, de niveaux silto-pélitiques d'âge thanétien à yprésien (Azmany et al., 1986). Le Lutétien est représenté à sa base par des alternances de niveaux à silex, de marnes et de calcaires peu phosphatés dans lesquels s'intercalent les derniers termes de la phosphatogenèse. La partie sommitale correspond à de puissants niveaux carbonatés, riches en gastéropodes, appelée dalle à Thersitées (Salvan, 1963).

D'un point de vue structural, la série phosphatée des Ouled Abdoun a la particularité d'être globalement peu affectée par des déformations imputables aux contraintes tectoniques du Crétacé supérieur et du Cénozoïque. Bien que les bordures du bassin de l'Oum Rabia aient toutes été soulevées et consécutivement érodées au cours de l'orogenèse alpine, il apparaît qu'aucune faille et qu'aucun basculement majeur ne décale notablement la régularité des reliefs de cuesta de cette région. Cependant, il doit être remarqué que les principaux oueds qui entaillent le plateau des phosphates et le drainent en direction du fleuve Oum Rabia ont une orientation qui coïncide avec celle des principales failles qui délimitent les grabens permo-triasiques affleurant au nord du bassin. Inscrite entre $\mathrm{N} 20^{\circ}$ et $\mathrm{N} 40^{\circ} \mathrm{E}$, cette orientation est aussi celle des failles qui ont contrôlé la sédimentation jurassique, puis la surrection cénozoïque du Moyen-Atlas (Fedan, 1989; El Hammichi et al., 2002). Il faut donc en déduire que ces failles anciennes existent sous la série sédimentaire des Ouled Abdoun et qu'elles ont pu rejouer sensiblement au cours de l'orogenèse Atlasique. Elles ont par exemple influencé la position et l'orientation des champs de diaclases affectant la dalle lutétienne et ont ainsi contrôlé la mise en place et le développement du réseau hydrographique actuel. Il est donc probable que certaines de ces failles hérités aient pu avoir une incidence sur l'épaisseur du Sénonien, et donc sur la distribution et l'épaisseur des couches de gypse dont la dissolution, facilitée par la circulation des fluides à travers ces failles, est à l'origine des dérangements de la série phosphatée.

\section{Evaporites du Sénonien moyen et phénomènes de dissolution}

Dans la majorité des bassins téthysiens et péritéthysiens, le Sénonien est généralement régressif, alors que le Maestrichtien est plutôt transgressif (Salvan \& Farkhany, 1982; Hardenbol et al., 1998). Dans les bassins intracontinentaux du Maroc, la série sédimentaire du Sénonien moyen (Santonien à Campanien) est couramment constituée de dépôts d'environnement marin très peu profond et confiné (Boujo, 1976). Les dépôts évaporitiques, en particulier gypseux, y sont fréquents. Ils ont été mentionnés et décrits dans de nombreuses régions du Maroc (Charroud, 1990; Daoudi, 1996; Daoudi \& Deconink, 1994; Daoudi et al., 1995; Dogan \& Özel, 2005).

Le Sénonien dans le bassin des Ouled Abdoun demeure très peu étudié étant donné que les couches sénoniennes se retrouvent à la limite inférieure de la série phosphatée, qu'elles sont dépourvues de phos- 


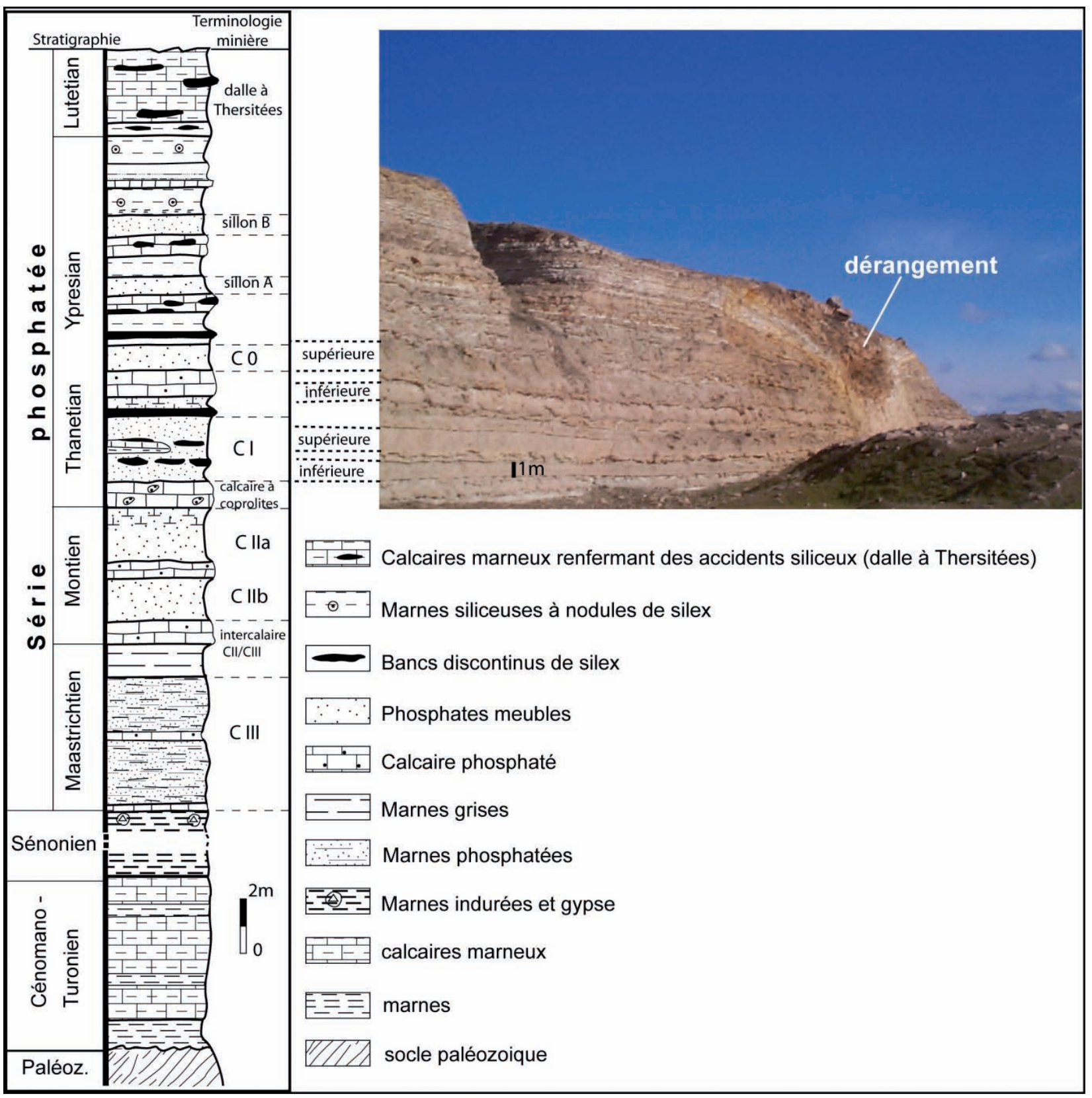

Fig. 2.-Colonne lithostratigraphique synthétique de la série phosphatée dans le bassin des Ouled Abdoun. La photographie, prise à Sidi Maati (localisation, Fig. 1a), montre la déformation des couches phosphatées au niveau des zones dérangées..

phates et donc sans grand intérêt économique. Les travaux de Choubert \& de Salvan (1976), ont pu décrire et séparer les éléments suivants dans le Crétacé supérieur de la région des Ouled Abdoun: (i) une série marneuse inférieur couleur grisâtre, jaunâtre par effet de l'altération à l'affleurement, (ii) des interbancs calcaires, par endroits marneux, avec localement des silicifications et (iii) une série marneuse supérieure, jaune d'or, avec parfois des niveaux marno-calcaires.

Le Sénonien, dans son ensemble, est décrit comme étant très peu fossilifère. Les quelques faunes qu'on a pu y retrouver n'apportent guère de précision d'ordre stratigraphique. Elles indiquent seulement qu'il s'agirait, a priori, d'un milieu peu propond (Choubert \& Salvan, 1976; Azmany, 1979; 

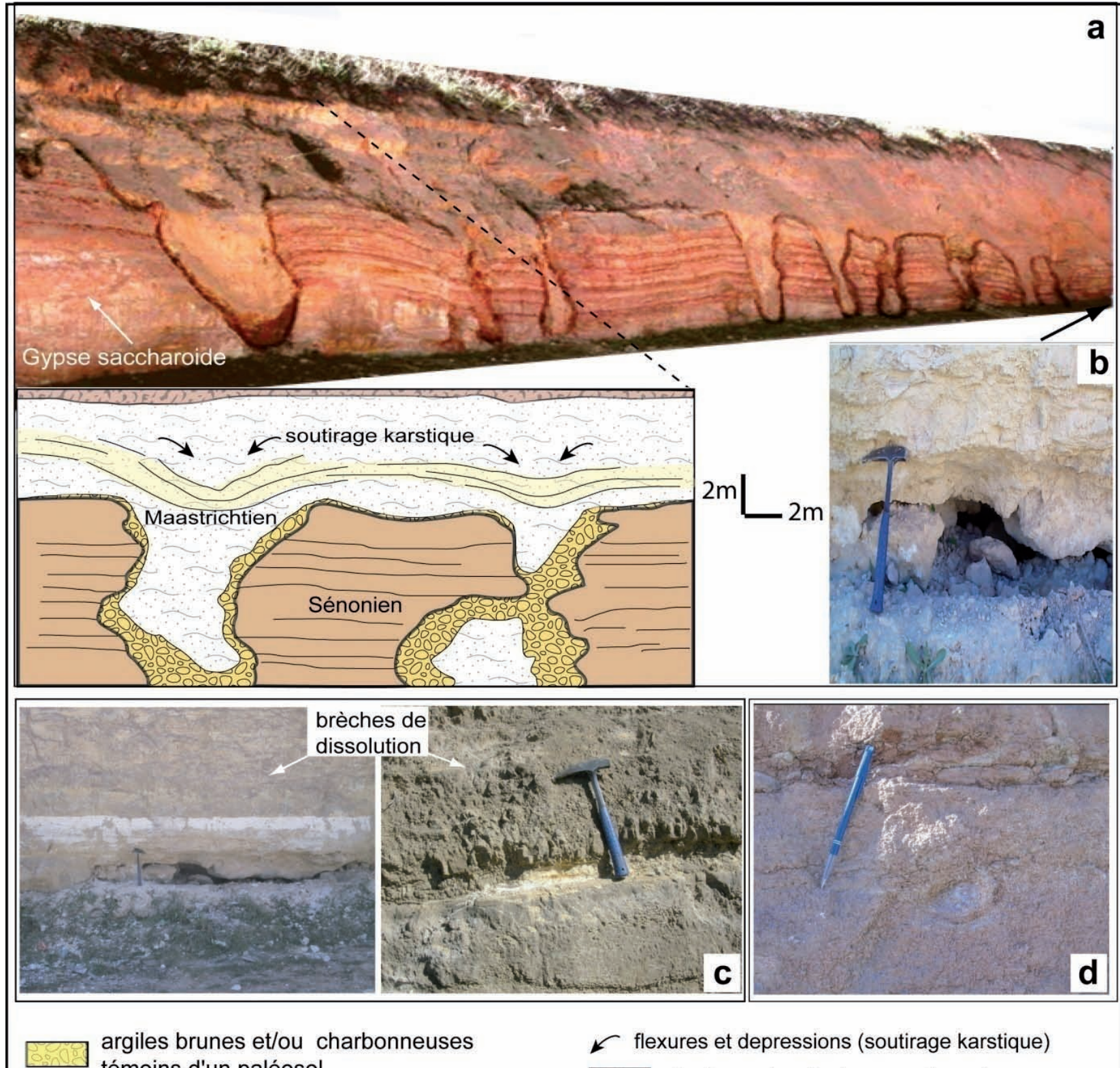

argiles brunes et/ou charbonneuses témoins d'un paléosol

$\leftarrow$ flexures et depressions (soutirage karstique)

$\sim$ surface de discontinuité

alluvions et colluvions quaternaires

marnes à gypse du Sénonien

\section{marnes phosphatés}

phospharénites d'âge maastrichtien

Fig. 3.-a. Cuvettes et puits de dissolution transperçant la série sénonienne dans le bassin d'Ouled Abdoun (Coupe Oued Zem, localisation Fig.1a). b. La dissolution dans une couche de gypse a créé une grande cavité non comblée. c. Faciès bréchiques au dessus des niveaux de gypse sccharoide. d. pseudomorphoses localisées d'anciennes évaporites.

Azmany et al., 1986). Les descriptions fournies par les auteurs ne mentionnent pas la présense de gypse ou d'autres minéraux évaporitiques dans la formation sénonienne. L'examen des affleurements séno- niens dans trois coupes situées sur une transversale NE-SW entre Oued Zem et Khouribga (localisation Fig. 1a), a montré autres faciès que ceux décrits par les auteurs. Dans cette région, le sommet des dépôts 


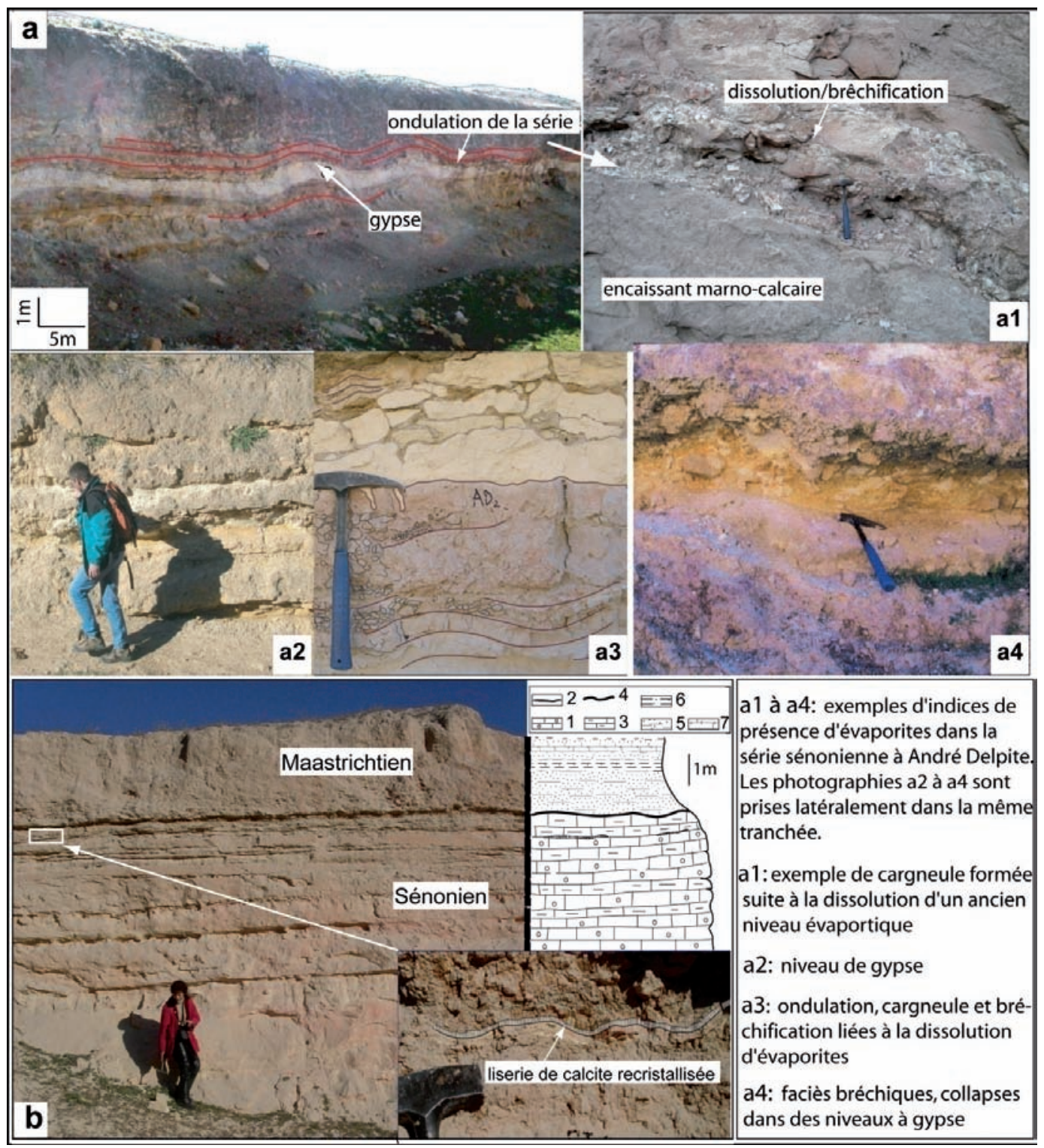

Fig. 4.-a. Coupe relevée à Andrée Delpite (localisation Fig. 1a) montrant des bancs de gypse inclus dans la série sénonienne et de nombreux indices de présence d'évaporites dans cette série (a1 à a4). b. Coupe Beni Iddir (localisation Fig. 1a). Le substratum sénonien est à dominance dolomicrite et montre parfois quelques liserés de calcite recristallisée probablement liées à des pseudomophoses calcitiques de fines couches de gypse. 1. Calcaire fossilifère 2. Liserée de calcite recristallisée 3. Calcaire marneux 4 . Discontinuité 5. Phospharénite 6. Marne phosphatée 7. Calcaire phosphaté.

traditionnellement attribués par les auteurs au Sénonien moyen, montre dans plusieurs sites des évidences d'évaporites et de figures sédimentaires et diagénétiques liées à la dissolution de sulfates. Nous avons identifié et décrit, pour la première fois, des couches de gypse saccharoide d'épaisseur centimétrique à métrique. Même quand le gypse et l'anhydrite ne sont pas présents (suite à des dissolutions), les indices de présence d'évaporites sont multiples: cavités de dissolution non comblées, cuvettes et puits de dissolution, structures de collapses, figures de bréchification et de pseudomorphose (Fig. $3 \& 4$ ).

Il doit être noté que les nombreuses et vastes mines à ciel ouvert actuellement ou récemment exploitées par l'OCP ne permettent pas d'observer le Sénonien car leur base se situe toujours au sein ou au toit du Maestrichtien, c'est-à-dire à la base des phos- 
phates exploitables. Les sites observés et échantillonnés sont tous des affleurements artificiels, formés soit par des talus de bord de route ou de voie ferrée, soit par d'anciennes carrières. Trois affleurements types, situés sur une transversale NE-SW entre Oued Zem et Khouribga (localisation Fig. 1a), sont brièvement décrits, dans cet article, car ils rendent compte de la variabilité faciologique du sommet du Sénonien moyen dans cette région.

\section{Coupe d'Oued Zem}

Située à la périphérie ouest de la ville d'Oued Zem, au niveau des tranchées récentes $\left(32^{\circ} 62^{\prime} 11^{\prime \prime} \mathrm{N}\right.$ $6^{\circ} 36^{\prime} 29^{\prime \prime} \mathrm{W}$ ) de la route R312 qui monte sur le plateau des phosphates, cette coupe (Fig.3a) montre des affleurements spectaculaires de dépôts évaporitiques. Il s'agit d'une formation de plus de $30 \mathrm{~m}$ de puissance et qui renferme des faciès évaporitiques à subévaporitiques où alternent marnes, calcaire, dolomicrite, marnes à évaporites et gypse. Le gypse de couleur blanche, est finement saccharoïde, pulvérulent. Il forme soit des bancs massifs d'épaisseur métrique, soit des niveaux centimétriques alternant avec des lamines stromatolithiques, des dolomicrites laminées et des marnes grises gypseuses appelés marnes grumeleuses par les géominiers de l'OCP. Les strates de gypse peuvent atteindre des épaisseurs de 3 mètres, plus particulièrement en sommet de série.

Dans les niveaux épais de gypse, les cavités de dissolution non comblées (Fig. 3b), de différentes dimensions, ont été fréquement relevées le long de la coupe étudiée. Les couches sus-jacentes aux bancs massifs gypseux montrent des brèches renfermant des fragments anguleux de micrite et de dolomicrite, cimentées soit par de la calcite ou soit par du gypse (Fig. 3c). Elles proviennent des phénomènes de dissolutions du gypse saccharoïde sous-jacent. Localement, les évaporites deviennent de plus en plus altérées et prennent des couleurs de plus en plus accusées, et ne subsistent qu'à l'état de pseudomorphoses localisées (nodules) (Fig. 3d).

Au sommet de la série sénonienne, le gypse est épais, lenticulaire. Les strates sont parfois tronquées, et se superposent de manière anormale. Il s'agit de plusieurs surfaces de discontinuités qui se télescopent les unes sur les autres décrivant des périodes d'arrêt de sédimentation. La géométrie des dépôts évaporitiques observés et l'épaisseur métrique des couches de gypse rappelle des dépôts de type «saline » (De Putter et al., 1994).
Dans cette série sénonienne, les discontinuités sont nombreuses et bien marquées. La plus spectaculaire se situe au sommet des dépôts attribués au Sénonien moyen. Elle correspond à une surface irrégulière d'érosion/alteration qui tronque les dépôts lagunaires évaporitiques et est scellée par les premiers dépôts grossiers, à fragments osseux, du Maestrichtien (Fig. 3a). De nombreuses formes karstiques sont observées, sous la forme de puits et de cuvettes de dissolution qui s'enfoncent dans la série sénonienne. La plupart des cuvettes ont un diamètre compris entre 1 et 3 mètres pour une profondeur de 0,5 à 2 mètres. Les puits présentent des diamètres comparables mais ils descendent pour la plupart jusqu'à 5 mètres de profondeur sous la base de la surface d'altération discontinue. A cette profondeur, ils se connectent à d'anciennes cavités stratiformes complètement effondrées, creusées au sein d'une épaisse couche de gypse. Les parois et les fonds des cuvettes et de certains puits sont généralement tapissés d'argiles brunes à verdâtres affectées par plusieurs générations de fentes de rétraction. Les fentes de rétraction les plus anciennes sont remplies par des argilites jaunes riches en petits fragments osseux et en quartz détritiques.

Des bioturbations ou traces de racines sont aussi observables au sein des remplissages argileux des cavités les moins profondes. Au fond de certaines cuvettes, des niveaux argileux noirâtres, à petits débris charbonneux, sont parfois interstratifiés dans les argiles brunes. Ce tapissage arileux de couleur rougeâtre, avec la présence de terriers et de traces de raciness, correspond à un paléosol développé sur substrat gypseux à la faveur d'une période d'émersion. Au dessus, les puits et cavités sont colmatés par les phospharénites du Maestrichtien. Ces dernières sont riches en quartz et contiennent des galets mous argileux (issus du remaniement des argiles sous-jacentes) et des fragments d'os de vertébrés. Ce remplissage phosphaté est en général très mal stratifié, probablement homogénéisé par la bioturbation. Les quelques litages qui peuvent encore y être observés indiquent des phénomènes de soutirages karstiques très probablement contemporains de la sédimentation maastrichtienne. D'autres puits, relevés dans cette coupe, montrent des figures de soutirages tardifs qui affectent non seulement les phospharénites maastrichtiennes mais aussi les niveaux phosphatés du Paléocène, des carbonates de l'Yprésien-Lutétien, voire des formations superficielles quaternaires. 

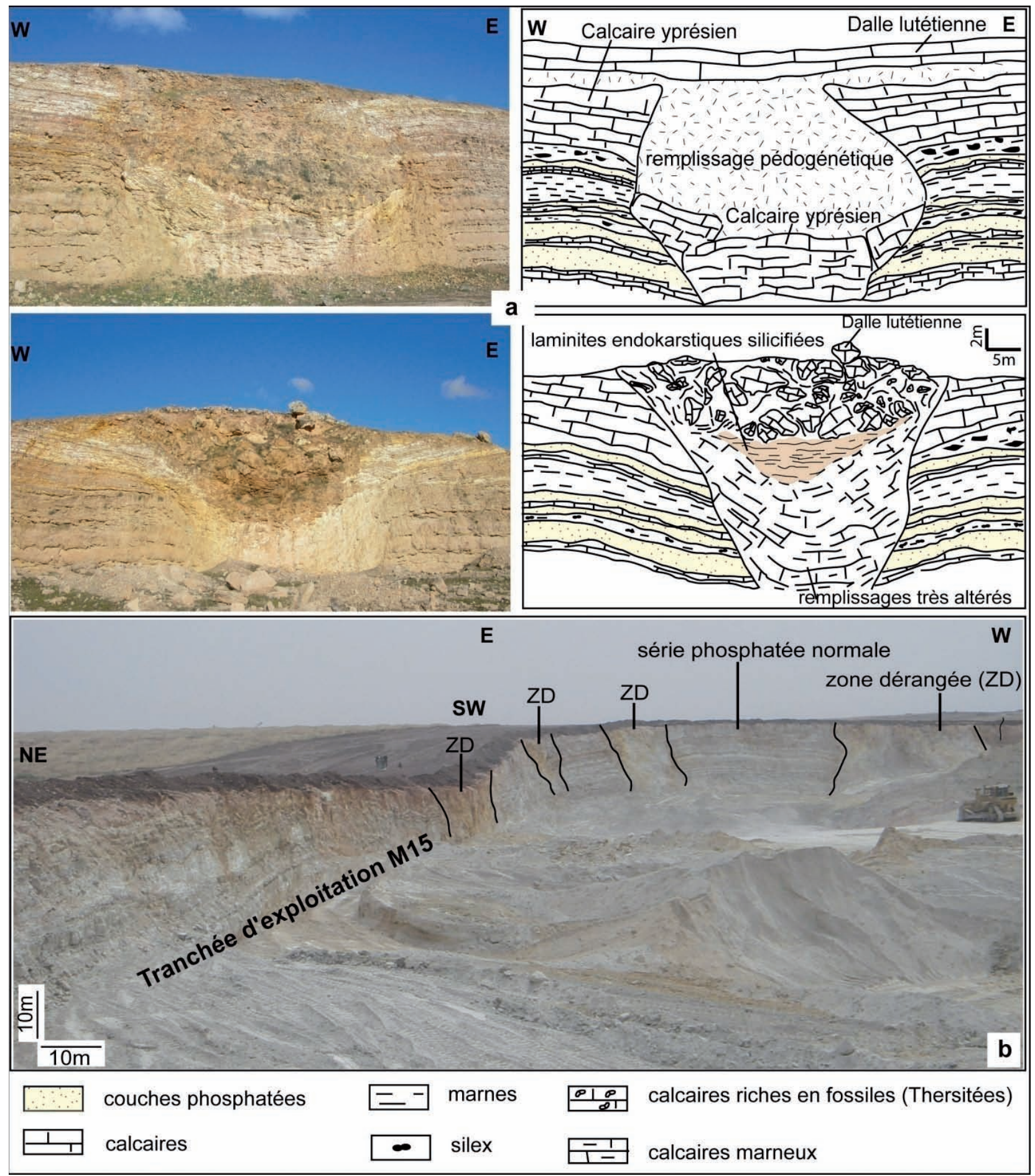

Fig. 5.-(a). Représentation schématique de deux types de fontis montrant des exemples de paléo-effondrement relevés dans la coupe de Sidi Maâti (localisation Fig. 1(a)). (b). Tranchée d'exploitation M15 à Sidi Chennane montrant des dérangements (ZD) affectant la série phosphatée. 


\section{Coupe André Delpite}

Située à environ $14 \mathrm{~km}$ au sud-ouest de la ville d'Oued Zem (localisation, Fig. 1a), cette coupe est formée par les tranchées d'une ancienne ligne de chemin de fer. Le Sénonien moyen y est principalement formé par des dépôts de milieu marin peu profond et ouvert, agité en alternance par une dynamique tidale ou par une dynamique de vagues. On y observe (Fig. 4a) une superposition des calcarénites fines structurées par des rides symétriques ou asymétriques, des calcarénites à litages horizontaux ou obliques à faible angle, des bancs de wackestones à gastéropodes et lamellibranches (souvent dolomitisés) et des interbancs de marnes grises.

Dans les derniers mètres de cette série apparaissent des faciès typiques de milieux marins plus restreints, parfois évaporitiques. Le gypse saccharoïde blanc y est présent, mais en faible quantité par rapport à Oued Zem. Les quelques bancs de gypse sont inclus dans une série bien stratifiée qui comporte surtout des stromatolites, des dolomies bréchiques, des marnes bréchiques et des cargneules (Fig. 4 a1 à a4). Les brèches et les cargneules sont ici typiquement liées à des dissolutions anciennes de niveaux de gypse. Le contact avec des fluides sous-saturés, dans un milieu peu profond et ouvert, provoque la dissolution des évaporites et crée l'espace à l'origine de la bréchification des roches sus-jacentes qui se fragmentent et se délitent, formant une brèche, d'effondrement connue, en anglais sous le nom de collapsebreccia (Orozco et al., 1999). Dans les marnes, les calcaires ou les dolomies, de fréquents nodules siliceux et/ou calcitiques suggèrent la présence d'anciens nodules de gypse ou d'anhydrite, pseudomorphosés. L'ensemble de ces structures indique un environnement de dépôt de tye sebkha.

Dans ce secteur, le contact avec les phospharénites du Maastrichtien n'est pas observable. La morphologie de la discontinuité sommitale, observée dans la coupe d'Oued Zem, demeure donc inconnue. Il faut toutefois remarquer que, comme à Oued Zem, des cavités stratiformes (maintenant effondrées) affectent les couches gypseuses et ont donc pu engendrer des soutirages affectant la géométrie des couches sus-jacentes. Les dérangements rencontrés dans les tranchées d'exploitation du phosphate du Grand Daoui, situé à $1 \mathrm{~km}$ d'André Delpite (localisation, Fig. 1a), témoignent de l'existence de ce phénomène de soutirage.

\section{Coupe de Beni Iddir}

Cette coupe est formée par les fronts de taille d'une ancienne carrière située à environ $6 \mathrm{~km}$ au sud de Khouribga (localisation, Fig. 1a). Elle permet d'observer dans de bonnes conditions le substrat des phospharénites du Maastrichtien. Ici, les dépôts attribués au Sénonien moyen sont exclusivement formés par des micrites et des dolomicrites stratiformes, à stromatolites et voiles algaires, contenant quelques fossiles dissous de gastéropodes et de lamellibranches. Issus de milieux lagunaires, voire lacustres, ces dépôts ne contiennent ni gypse, ni indices de la présence ancienne de sulfates ou de halite. Toutefois, de rares liserés ondulants de calcite fibreuse, finement recristallisés, ont été observés à la base de la série maastrichtienne (Fig. 4b). Ce type de recristallisation peut être apparenté à des pseudomorphoses calcitiques d'une fine couche de gypse, probablement discontinue, intercalée entre les dépôts carbonatés et phosphatés. Cet hypothétique phénomène diagénétique est habituel et fréquemment rencontré dans ce type d'environnement (Arenas et al., 1999).

L'équivalent de la discontinuité sommitale, observée à Oued Zem, est ici une surface subhorizontale, plane et non durcie, qui matérialise un contact brutal entre des dolomicrites et les premiers dépôts phosphatés riches en ossements grossiers du Maastrichtien. Des processus de ravinement en milieu sousmarin agité sont vraisemblablement responsables du développement d'une telle discontinuité lors de la transgression maestrichtienne.

\section{Description des dérangements de la série phosphatée}

Au sein de la série phosphatée, les déformations les plus fréquentes et les plus spectaculaires correspondent à des fontis (Fig. 5), c'est-à-dire à des éboulements et remplissages per descensum engendrés par l'effondrement de cavités sous-jacentes. De forme généralement cylindrique, avec des parois verticales ou très inclinées (parfois surplombantes), ces fontis ont un diamètre compris entre 5 et 150 mètres. Ils traversent habituellement la totalité de la série phosphatée (environ 50 mètres) et se propagent per ascensum jusqu'aux calcaires sus-jacents de la Dalle à Thersitées d'âge lutétien.

Du fait de leur remplissage systématique, ces fontis sont très difficilement perceptibles depuis la surfa- 


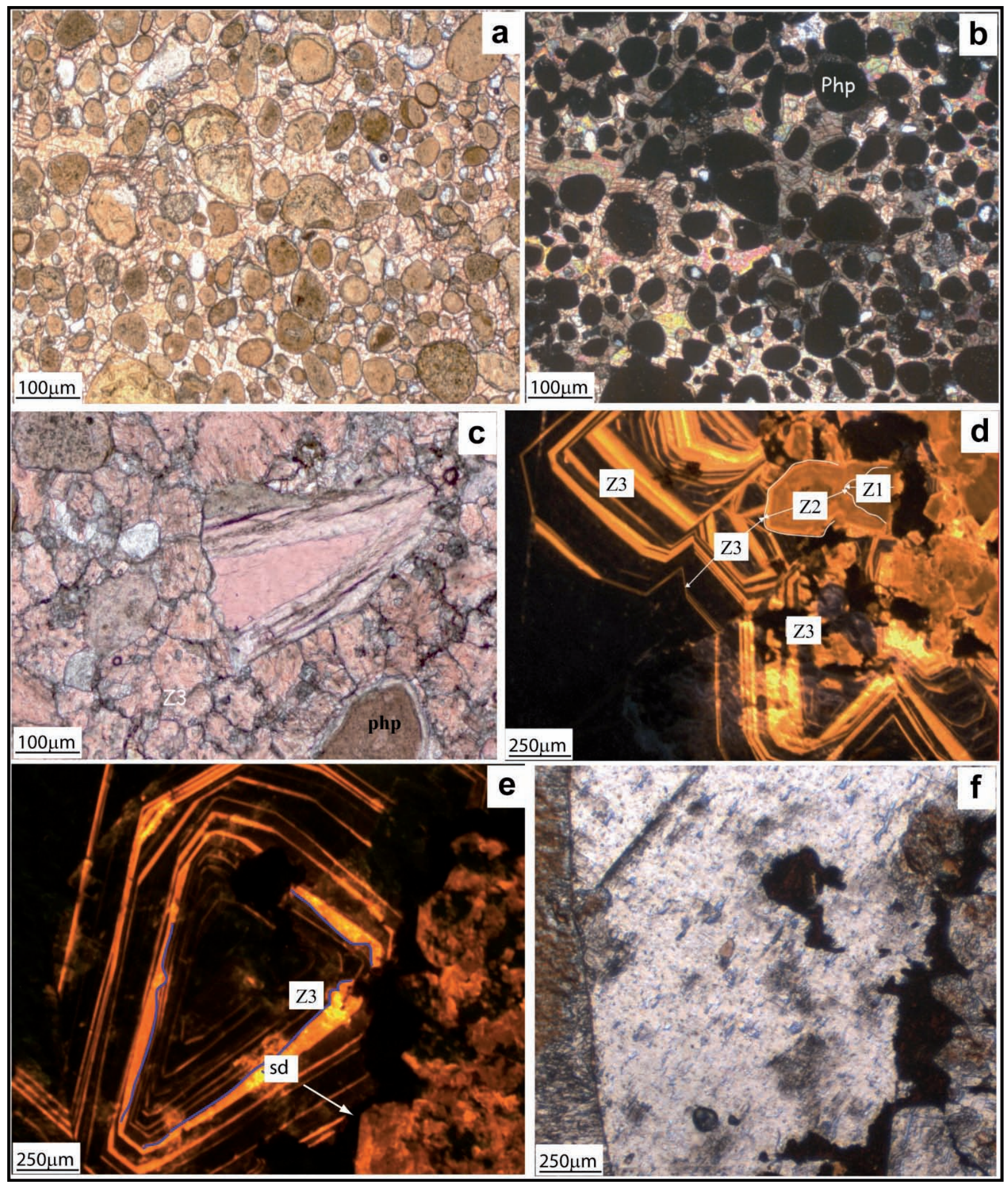

Fig. 6.-a et b. Grains phosphatés arrondis baignant dans une matrice calcitique. Certains grains sont plus ou moins corrodés. On peut percevoir que le contour des phosphates est parfois totalement calcitisés. c. Apatite osseuse d'un reste organique. d. Différentes générations de ciments sparitique Z1: Sparite faiblement luminescente Z2: Sparite moyennement luminescente Z3: Sparite alternant de larges bandes non luminescentes et d'étroites bandes fortement luminescentes. e et f. Figures de dissolution intra-zones. Des golfes de corrosion se dessinent entre deux bandes d'une même sparite. php: grain de phosphate, Sd: surface de dissolution. 
ce. Leur détection peut néanmoins être assurée par des sondages mécaniques (à maille très serrée), par des méthodes géophysiques (Kchikach et al., 2002; Kchikach et al., 2006) ou par l'analyse de certains clichés aériens révélant une croissance différentielle de la végétation à leur aplomb. Le remplissage peut varier d'un fontis à l'autre. Globalement, il comporte toujours des blocs constitués de sédiments encore stratifiés provenant de l'effondrement des parois de l'encaissant. Entre les blocs, le liant est formé, suivant les cas, par des argiles brunes, par des marnes grises siliceuses, par des marnes phosphatées, par des phospharénites meubles ou plus rarement par des spéléothèmes calcitiques ou siliceux. Dans certains fontis, l'affaissement a conservé l'ordre et l'agencement général des couches de l'encaissant. Ainsi, dans les cas illustrés sur la figure $5 \mathrm{a}$, les strates yprésiennes et lutétiennes sont clairement identifiables; elles sont fracturées, déformées et légèrement basculées, mais elles ont conservé leur position stratigraphique et une partie de leur stratonomie bien qu'étant affaissées d'une dizaine de mètres par rapport à leur situation initiale. D'autres fontis montrent un remplissage moins stratifié, mais il est toujours possible d'y observer des blocs plus ou moins altérés provenant du démantèlement des calcaires yprésiens ou lutétiens.

Les bancs marno-calcaires et phosphatés formant la paroi des fontis sont parfois affectés par des déformations qui consistent soit en amincissements et flexures de strates, soit en basculements par pans entiers. Les amincissements affectent préférentiellement les faciès meubles: marnes et phospharénites. Ils s'expliquent par le fluage horizontal des sédiments non indurés en direction des fontis et donc par la diminution du volume de ces strates. Ce fluage horizontal provoque une flexuration progressive des strates, qui peut rayonner jusqu'à une dizaine de mètres autour des parois du fontis, formant ainsi une doline périphérique évasée. En surface, cette doline est masquée car comblée et nivelée par des remplissages anciens ou par des formations superficielles. Les basculements par pans entiers sont délimités par des fractures de compaction différentielle, par des fractures de décollement et parfois par des fontis dissymétriques. Pouvant affecter des surfaces relativement vastes (jusqu'à $10.000 \mathrm{~m} 2$ ), ces basculements montrent que des pans entiers de la série déjà indurée ont pu s'affaisser et basculer sous l'effet de l'effondrement de vastes cavités stratiformes situées juste sous la série phosphatée.
A Sidi Maati (Fig. 5a), sur le bord de certains fontis, au dessus de la formation yprésienne, on peut notamment identifier un profil d'altération pédologique. Les faciès bruns très altérés formés essentiellement d'arigles à traces de raciness et de galets mous témoignent probablement d'une période d'émersion locale. Au final, les calcaires lutétiens scellent cet ensemble et ne sont que très peu flexurés.

Dans la mine à ciel ouvert de Sidi Chennane (localisation, Fig. 1a), les fontis (derangements) sont très fréquents (Fig. 5b) et perturbent sérieusement les travaux d'extraction des phosphates dans les chantiers d'exploitation. Les coupes géologiques que nous avons réalisées sur les parements des tranchées d'exploitation nous ont permis de décrire trois types de fontis, basés sur la nature des matériaux qui les remplissent. Le type I, le plus fréquent, est formé exclusivement de calcaire silicifié. Il montre bien le contraste pétrographique et, par conséquent rhéologique, qui existe entre la série phosphatée normale, formée de niveaux phosphatés et marno-calcaires, et la masse homogène de calcaire silicifié à nodules de silex. Le type II est constitué d'un mélange de blocs de calcaires à gros nodules de silex, de marnes, d'argiles et de portions de bancs de silex. Dans le type III, on retrouve ces mêmes constituants mais avec une nette prédominance des marnes siliceuses.

\section{Description microscopique des dérangements et leur voisinage immédiat}

Parallèlement à la description macroscopique de la série évaporitique sénonienne, des phénomènes de dissolution, pseudomorphose et processus épikarstique et endokarstique, en particulier au voisinage immédiat des zones dérangées, une étude microscopique sur une trentaine d'échantillons prélevés dans les différents sites étudiés a été menée. Ces échantillons ont essentiellement ciblé les parois des effondrements, le cœur de ces structures et la série phosphatée non perturbée. L'objectif de cette étude est de caractériser les phénomènes postérieurs et antérieurs à la phosphatogenèse ainsi que d'identifier les différents ciments calcitiques et leurs origines. Aussi, elle cible la détection de traces de dissolutions et leur possible confrontation avec les phénomènes observés à plus grande échelle. Pour ce faire, une analyse microscopique; a été réalisée sur trente trois lames colorées à l'alizarine-ferricyanure de potassium. Douze d'entre elle ont été observées en cathodoluminescence à l'aide d'une caméra numérique haute 
résolution. Dans ce qui suit, on décrit et on interprète la signification des différentes phases minérales observées.

\section{Phosphates et ciments phosphatés}

Les phosphates marocains sont des phosphates marins formés sur une plate forme continentale, sur substrat silto-sableux. Le climat a été défini comme étant chaud, dans une mer présentant un chimisme particulier, et où sont réuni toutes les conditions de phosphatogenèse (Lucas et al., 1979). En lame mince (Fig. 6 a, b \& c) ces phosphates se présentent sous diverses formes: en grains, en fragments squelettiques ou encore sous forme de ciments. Les grains phosphatés sont arrondis, ovoïdes, ou irrégulièrs, noirâtres obscuries par l'imprégnation de la matière organique. Leur état de conservation est généralement bon, et des petites plages de calcites y sont parfois incluses. L'apatite peut se présenter sous forme de débris organiques (dents, écailles de poissons), disséminés dans la trame.

Les faciès des Ouled Abdoun montrent des ciments de calcite sparitique, se mettant en place entre grains phosphatés ou en remplissage de fractures. La calcite forme un ciment esquilleux qui réunit les grains phosphatés. On peut observer en lames minces que cette calcite non seulement remplie l'espace disponible, mais aussi s'attaque aux grains d'apatite par l'extérieur en les corrodant. Les liserés calcitiques marquent entre nicols croisés la limite entre apatite et calcite.

En cathodoluminescence, ces sparites, révèlent des bandes concentriques nettes qui peuvent être regroupées en trois principales zones de luminescences (Fig. 6 d).Une première zone (Z1), très faiblement luminescente, sans zonation interne distincte, de teinte brun foncé. Une deuxième zone (Z2) moyennement luminescente, se compose d'une alternance de bandes brunes à orangées faiblement contrastées. Enfin, une troisième zone (Z3), caractérisée par une alternance de larges bandes non luminescentes et de fines bandes jaunes fortement luminescentes dont les limites sont souvent brutales et nettes. Cette dernière zone est généralement la plus développée, la plus épaisse, dans les pores de grande taille. Ces trois grandes générations de sparites, se retrouvent dans environ toutes les lames analysées et toujours dans le même ordre chronologique (Z1, Z2, Z3).
Les luminescences sont directement liées aux taux d'insertion de $\mathrm{Mn}^{2}+$, et $\mathrm{Fe}^{2}+$ dans le réseau cristallin et sont le reflet de la croissance des minéraux carbonatés et traduisent des changements du chimisme et des renouvellements de fluides de précipitation (Machel, 1985; Emery et Dickson, 1989; Durlet et al., 1992). Les ciments calcitiques décrits dans les sédiments phosphatés du bassin des Ouled Abdoun, peuvent avoir diverses origines. Les plus tardifs peuvent probablement avoir une origine météorique (Z3). Des ciments similaires ont été décrit comme tels par plusieurs auteurs (Emery \& Dickson 1989; Cazenave et al., 2003; Chapoulie et al., 2005). On ne peut cependant pas exclure que ces sparites puissent être d'origine marine. Des mesures isotopiques peuvent être très porteuses pour une détermination plus rigoureuse de leurs occurrences.

La croissance cristalline de ciments sparitiques est parfois interrompue. Au sein des cristaux, la limite entre deux bandes successives (luminescente et non luminescente) peut parfois décrire une surface sinueuse, irrégulière, cicatrisée par les bandes suivantes (Fig. 6 e \& f). Ces figures, couramment indiqués comme étant des golfes de corrosion, sont caractéristiques de la dissolution au sein de la calcite (Mazullo et al., 1990; Braithwaite et al., 1992). Les golfes de corrosion entaillent profondément la Z1, et sont rapidement scellés par de la calcite fortement luminescente de la Z2. La zone 1 est par endroit absente, elle n'a donc pas précipité ou a disparu complètement sous l'effet de la dissolution.

Les phases de croissance sparitiques traduisent des milieux sursaturés, tandis que les phases de dissolution indiquent l'invasion d'eaux plus agressives (sous saturées). Ces dernières correspondent à des périodes de recharge et de renouvellement de l'eau interstitielle par les pluies (Chapoulie et al., 2005). Les ciments observés ici n'ont définitivement pas les caractéristiques d'une sparite d'enfouissement. La série phosphatée du bassin des Ouled Abdoun n'a pas ou a peu été sujette à des phénomènes d'enfouissement accusés (Boujo, 1976). L'absence de stylolites, de figures de desquamation et de ciments ferreux, sont quelques caractères appuyant cette hypothèse. Les sparites sont notamment peu ferreuses, et dessinent des bandes concentriques très contrastées relativement complexes. Ces ciments sont probablement d'origine météoriques, le calcium étant alors issu des lessivages karstiques et /ou pédogénétiques ayant lieu dans le bassin des Ouled Abdoun. 


\section{Dolomite et dédolomitisation}

La plupart des lames minces examinées montrent que les faciès dans le bassin des Ouled Abdoun sont affectés ou ont été affectés par une intense dolomitisation matérialisée par des rhomboèdres à section losangique. Ils sont parfois nombreux, présentant des tailles diverses, et tapissant tout l'espace disponible entre grains phosphatés. Souvent, ces rhomboèdres sont partiellement voire totalement dédolomitisés (Fig. 7 a, b, c \& d). Dans les grands rhomboèdres (Fig. 7a), la dolomie a été remplacée par de la sparite appartenant aux zones 2 et 3 , décrivant ainsi un processus de dissolution/remplacement.

La calcite observée ici n'est parfois pas considérée comme un ciment sparitique mais plutôt comme une microfabrique de remplacement. En effet, les sparites sont secondaires, elles remplacent les dolomites, préservant leur forme initiale. Les dolomites communes aux environnements peu profonds, fréquemment en relation avec les phénomènes d'évaporation intense (Machel, 198; Arenas et al., 1999), auraient pu provenir d'une forte concentration de leur composantes dans les eaux lors de la précipitation en tant que précipité primaire, remplacé ensuite pendant les étapes précoces de la diagenèse. Les phénomènes de dédolomitisation indiquent indéniablement que des eaux agressives aient pu circuler dans les dépôts évaporitiques et sub-évaporitiques du bassin des Ouled Abdoun. Etant donné le caractère tardif de ces dissolutions (remplacement par de la sparite zonée), l'hypothèse la plus plausible pour décrire l'origine de ces phénomènes, serait la circulation d'eau météorique. L'âge de ces manifestations peut probablement être celui de la mise en place des surfaces paléokarstiques décrites ci-dessous (cf. partie discussion).

\section{Sulfates et silicates}

Plusieurs ciments anhydritiques sont aussi reconnus dans les lames minces, et s'installent entre les grains phosphatés, ou précipitent dans les fractures. Quelques cristaux de gypse peuvent se retrouver préservés à l'état primaire, bien cristallisés, laissant apparaître deux grandes familles de clivages (Fig. 7e).

L'abondance de la silice dans la série phosphatée du bassin des Ouled Abdoun est un carctère très général. Elle s'observe sous diverses formes: en nodules, en bancs continus, ou encore en formations discontinues à caractère lenticulaire, au sein d'autres couches (Salvan, 1982; Boujo, 1976). Elle peut aussi avoir épigénisé les débris squeletiques. Elle présente des teintes de biréfringence assez élevées et n'est absolument pas luminescente. On reconnaît notamment au niveau des lames minces des sphérulites sous forme de calcédoine fibreuse et radiaire (Fig. 7f), celle-ci contient notamment des inclusions de gypse, témoignant des phénomènes de remplacement qui ont pu avoir lieu. De telles figures de pseudomorphoses siliceuses de gypse ont été observées dans plusieurs lames étudiées. Les phénomènes de dissolution, recristallisation, remplacement de sulfates en particulier de gypse et d'anhydrite, sont connus comme étant commun aux environnements supratidaux (Elorza \& Rodriguez-Lazaro, 1984) et aux environnements évaporitiques continentaux (Salvany et al., 1994).

L'anhydrite observée est secondaire et provient de la déshydratation du gypse. Sa présence indique clairement un milieu marin peu profond et une forte concentration en sulfates des eaux interstitielles. Les mélanges entre eaux interstitielles et eaux météoriques vont engendrer la modification $\mathrm{du} \mathrm{pH}$ des eaux de diagenèse. Ces eaux vont être responsables de la dissolution initiale des sulfates et de la formation des premières cavités et effondrements (collapses). La variation du $\mathrm{pH}$, suite à l'introduction des eaux météoriques rend les dolomites instables qui laissent place aux dédolomites observées dans plusieurs lames examinées.

\section{Discussion: origine et âge des fontis}

La distribution spatiale des fontis affectant la série phosphatée montre un lien étroit avec la distribution du gypse du sommet du Sénonien moyen. Dans la région d'Oued Zem, où les couches de gypse sont épaisses, les fontis sont nombreux. Plus à l'Ouest, dans les tranchées d'exploitation des phosphates à Sidi Daoui (Fig.1a) où les couches de gypse sont moins développées, les fontis existent mais sont plus rares et plus petits. Encore plus à l'ouest, dans les secteurs de Khouribga et Beni Iddir, les fontis sont absents (ou rarissimes) de même que les niveaux de gypse.

Dans les mines à ciel ouvert implantées au dessus des couches de gypse, les fontis peuvent perturber jusqu'à 20\% de la surface exploitée. Dans la découverte de Sidi Chennane par exemple (Fig. 5b, localisation Fig.1 a), les fontis sont très fréquents et leur 


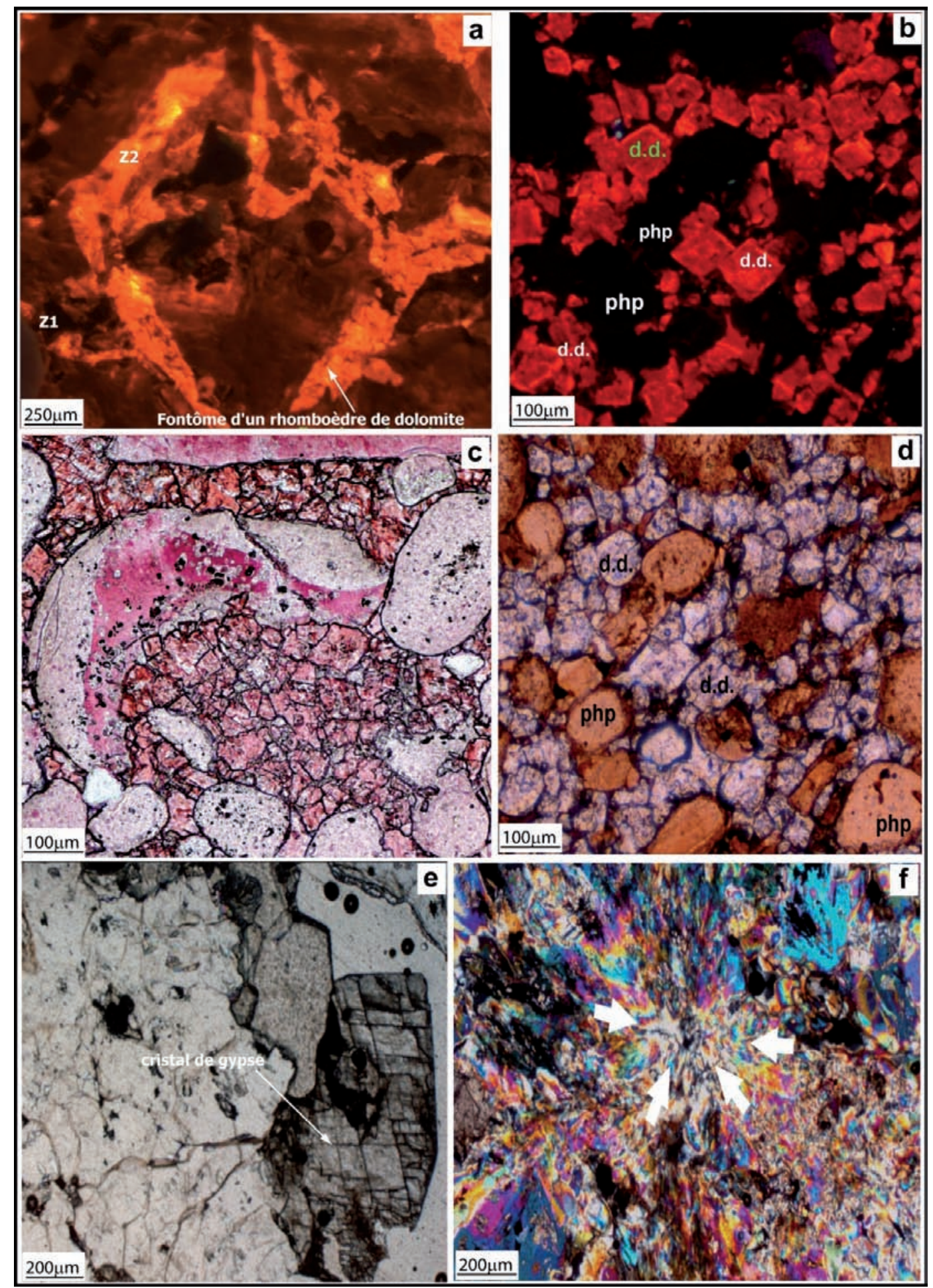

Fig. 7.- -a. Fontôme d'un rhomboèdre de dolomite maintenant dédolomitisé et remplacé par de la sparite moyennement luminescente (Z1-Z2). b et d. Sparites (observés en Cathodoluminescence), ayant conservés la section losangique des rhomboèdres initiaux. Les cristaux présentent une luminescence faible à moyenne (Z1-Z2) et s'indentent les uns les autres.c. Rhomboèdres calcitiques dédolomitisés, colorés en rose par l'alizarine ferricyanure de potassium. e. Cristal de gypse à clivage parfait, au centre d'un nodule siliceux, témoin probable du remplacement ayant eu lieu suite à la circulation d'eaux météoriques. f. Pseudomorphose siliceuse d'anhydrite. Cliché effectué dans un nodule de silice à croissance centripète. php: grain de phosphate, dd: dédolomitisation. 
distribution spaciale n'apparait pas suivre une direction priviligiée (Kchikach et al., 2006). Une telle répartition des fontis n'est pas compatible avec l'effondrement de salles ou de galeries karstiques linéaires classiquement développées dans les karsts calcaires comme le propose El Felah (1990). Les fontis observés à Oued Zem ou dans les découvertes à ciel ouvert des phosphates (Sidi Chennane, Sidi Maati et Sidi Daoui), ne sont pas alignés selon une direction priviligiée qui pourrait être attribuée à une structuration régionale majeure.Cette distribution indique au contraire que le gypse sous jacent aux couches de phosphate exploité, du fait de sa forte solubilité, est criblé de cavités de dissolutions distribuées en un maillage complexe probablement contrôlé par le réseau de fractures locale qui devrait avoir joué un rôle précurseur de la dissolution du gypse en favorisant les infiltrations localisées des eaux météoriques.

Plusieurs auteurs (Klimchouk, 1996; Elorza \& Santololla, 1998; Rodriguez-Aranda et al., 2002; Dogan \& Ozel, 2005) décrivent de tel criblage de cavité et de fontis dans de nombreuses autres régions du monde où des couches de gypses se retrouvent enfouies à quelques mètres ou dizaines de mètres sous la surface. Les observations effectuées dans ces diverses régions montrent qu'un criblage de fontis ne peut se développer que si les trois conditions suivantes sont réunies: (i) le gypse sous-jacent doit avoir (ou avoir eu) une épaisseur plurimétrique; (ii) il doit avoir été en contact à une ou plusieurs reprises avec des circulations d'eaux météoriques, sous-saturées et (iii) il doit subsister des zones de gypse non dissoutes entre les cavités effondrées.

Dans le cas du basin des Ouled Abdoun, il est clair que ces conditions furent réunies dans les secteurs où les fontis sont très nombreux: le gypse y est encore présent en bancs pluri-décimétriques à plurimétriques et il est parcouru par de nombreuses cavités généralement effondrées et colmatées. Il reste à discuter l'âge des dissolutions de ce gypse et donc de la chronologie des circulations météoriques.

Les reconstitutions paléogéographiques établies par plusieurs auteurs (Choubert \& Salvan, 1949; Ambroggi, 1963; Duffaud et al., 1966; Boujo, 1976; Amrhar, 1995; Le Roy, 1997) décrivent plusieurs périodes d'émersion dans le Maroc atlantique depuis le Sénonien. Des mouvements épirogéniques ont déclenché à la fin du Santonien une regression régionale dans tout le bassin atlantique marocain dont fait partie le bassin des Ouled Abdoun. Cette régression régionale se manifeste dans les bassins crétacés atla- siques par des surfaces d'émersions dans le Moyen atlas (Charroud, 1990; Andreu \& Tronchetti, 1994) et des exhumations locales dans le haut Atlas (Laville, 1985; Laville \& Piqué, 1992; Piqué et al., 1998). Ce phénomène est général et se manifeste lors de la régression décrite dans le bassin des Oulad Abdoun (Ambroggi, 1963) par des émersions locales qui ont permit une karstification intense de la série sénonienne qui accompagne une diminution progressive de l'extension du bassin, comme d'ailleurs tous les bassins atlantique marocain à cette période (Wiedmann et al., 1982; Le Roy, 1997).

Les travaux de Huvelin (1973) montrent que les facies à Thersitées, qui coiffent le complexe phosphaté dans le bassin des Ouled Abdoun, sont remplacés à l'Est par des calcaires à Huîtres ou Turritelles. Plus à l'Est encore, dans les piemonts de l'Atlas, on passe aux faciès lacustres commençant parfois dès l'Eocène inférieur. Ces dépôts annoncent en quelque sorte la régression générale qui suit l'Eocène moyen et introduit le cycle des mouvements alpins paroxysmaux (Michard, 1976 \& Piqué et al., 2007). Dans ce qui suit, on essaiera de placer nos observations de terrain dans ce cadre regional pour expliquer le processus précurseur de la dissolution du gypse sénonien, le développement et la maturation des profils karstiques et par suite le mode de genèse des fontis qui existent actuellement dans les tranchées d'exploitation des phosphates.

\section{Dissolutions sénoniennes}

Les puits karstiques de la région de Oued Zem, se sculptent dans un paléosol épais de quelques mètres. La coupe examinée montre que la discontinuité qui forme le toit de la série sénonienne (Fig. 8) peut être interprétée comme un épikarst sur substrat gypseux. Elle est en effet criblée de cuvettes et de puits de dissolution qui sont scellées par les premières phospharénites du Maestrichtien. Des argiles brunes, localement rubéfiées ou charbonneuses, y témoignent du développement d'un paléosol. Ce paléosol est souvent déstructuré car affecté à la fois par des remaniements sédimentaires (galets mous argileux) survenus au début de la transgression maestrichtienne et par des soutirages karstiques plus tardifs. La présence de puits cylindriques est parfois interprétée par les racines d'arbres et/ou de buissons qui prolifèrent dans les zones desséchées. Ces racines forment des voies préférentielles de percolations d'eaux météoriques, s'élargissant avec la dissolution du gypse. Il 

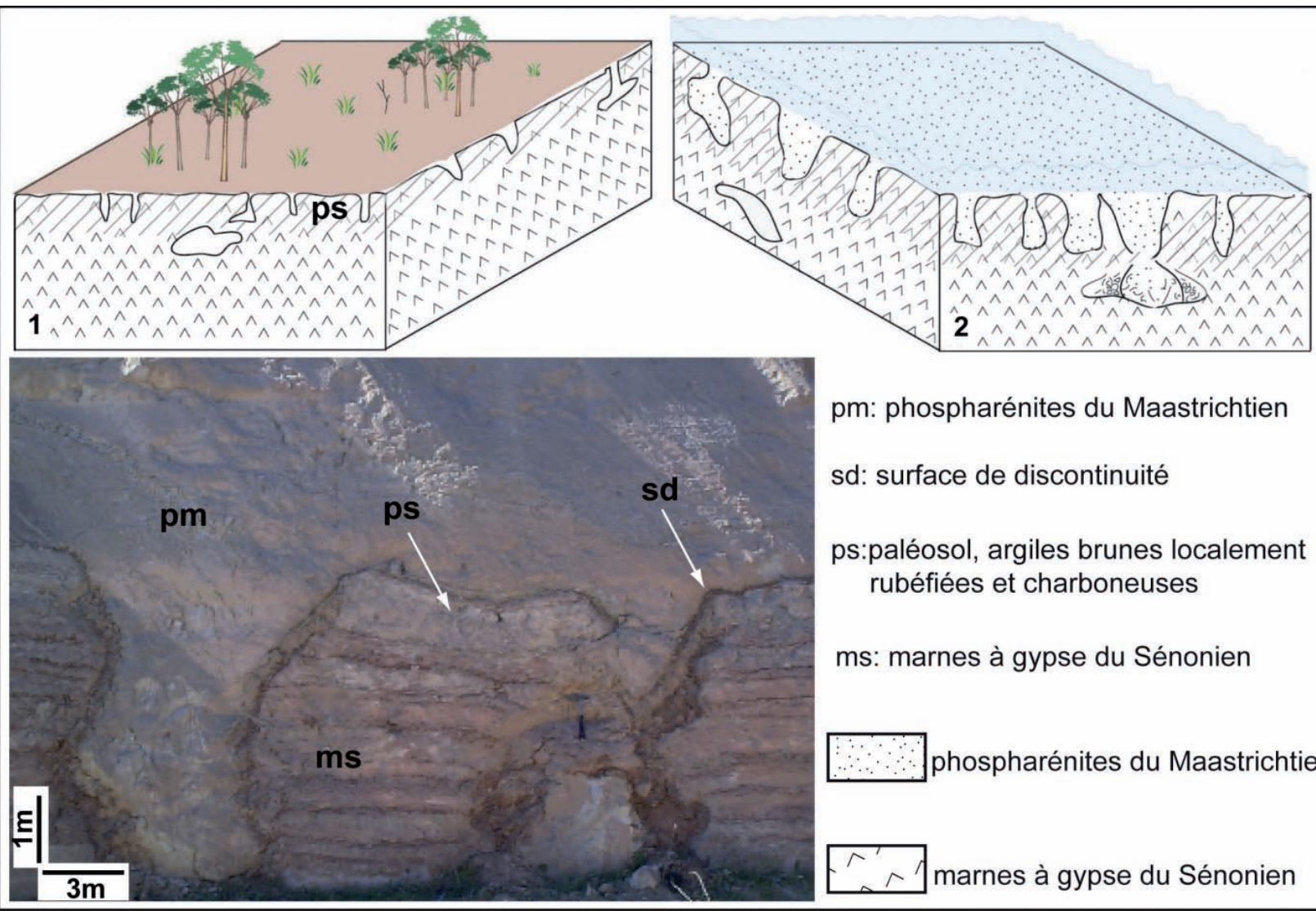

pm: phospharénites du Maastrichtien

sd: surface de discontinuité

ps:paléosol, argiles brunes localement rubéfiées et charboneuses

ms: marnes à gypse du Sénonien

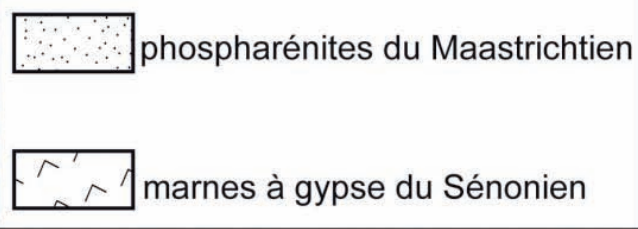

Fig. 8.-Modèle de l'évolution chronologique du développement des karsts sur substrat gypseux, les depressions karstiques comblées par les phospharénites du Maastrichtien et le palésol est coincé entre les deux formations (coupe Oued Zem, localisation Fig. 1a).

s'en suit alors la formation de puits et de conduits qui vont être connectés avec la surface épikarstique (Rodriguez-Aranda et al., 2002).

Le développement de paléosols et de surfaces de discontinuités à la fin du Sénonien à Oued Zem et à Beni Iddir, peut être facilement intégré dans le contexte paléogéographique régional à cette époque. Il serait lié à l'émersion fini-sénonienne indiquée par les reconstitutions paléogéographiques citées ci-dessus. Au Sénonien, on assiste dans un premier stade, à l'accumulation des dépôts de gypse dans un milieu marin peu profond (Fig. 8(1)). Après le retrait de la mer, dans un second stade, le gypse induré est colonisé par les plantes, il s'en suit la formation d'un paléosol sur les résidus de dissolution. La présence très probable de plantes aurait engendré la formation de conduits initiaux sous l'action des eaux météoriques. L'élargissement des cavités karstiques par le biais de la dissolution des conduits initiaux induit la formation de dépressions sous-jacentes dont les morphologies sont diverses. Ces depressions seront rem- plies par des phospharénites à la faveur de la transgression maastrichtienne (Fig. 8(2)). En s'amalgamant, les depressions peuvent engendrer la formation de paléoeffondrements sous couverture sédimentaire. Les enracinements de ces paléoeffondrements sont identifiés sur la coupe de Oued Zem.

\section{Karstification yprésienne et/ou lutétienne}

Lorsqu'elle peut être observée, la discontinuité située à l'interface entre les niveaux calcaréomarno-dolomitiques attribués à l'Yprésien et les dépôts calcaires de la dalle à Thersitées attribués au Lutétien est couramment soulignée par un ou plusieurs paléosols superposés (Fig. 9). La pédogenèse y est matérialisée par des profils d'altération argileux et par des traces de racines. Des ciments micritiques formant des calcrètes laminés, flexueux et peu épais (quelques millimètres) peuvent ponctuellement y être observés. A l'aplomb de certains fon- 


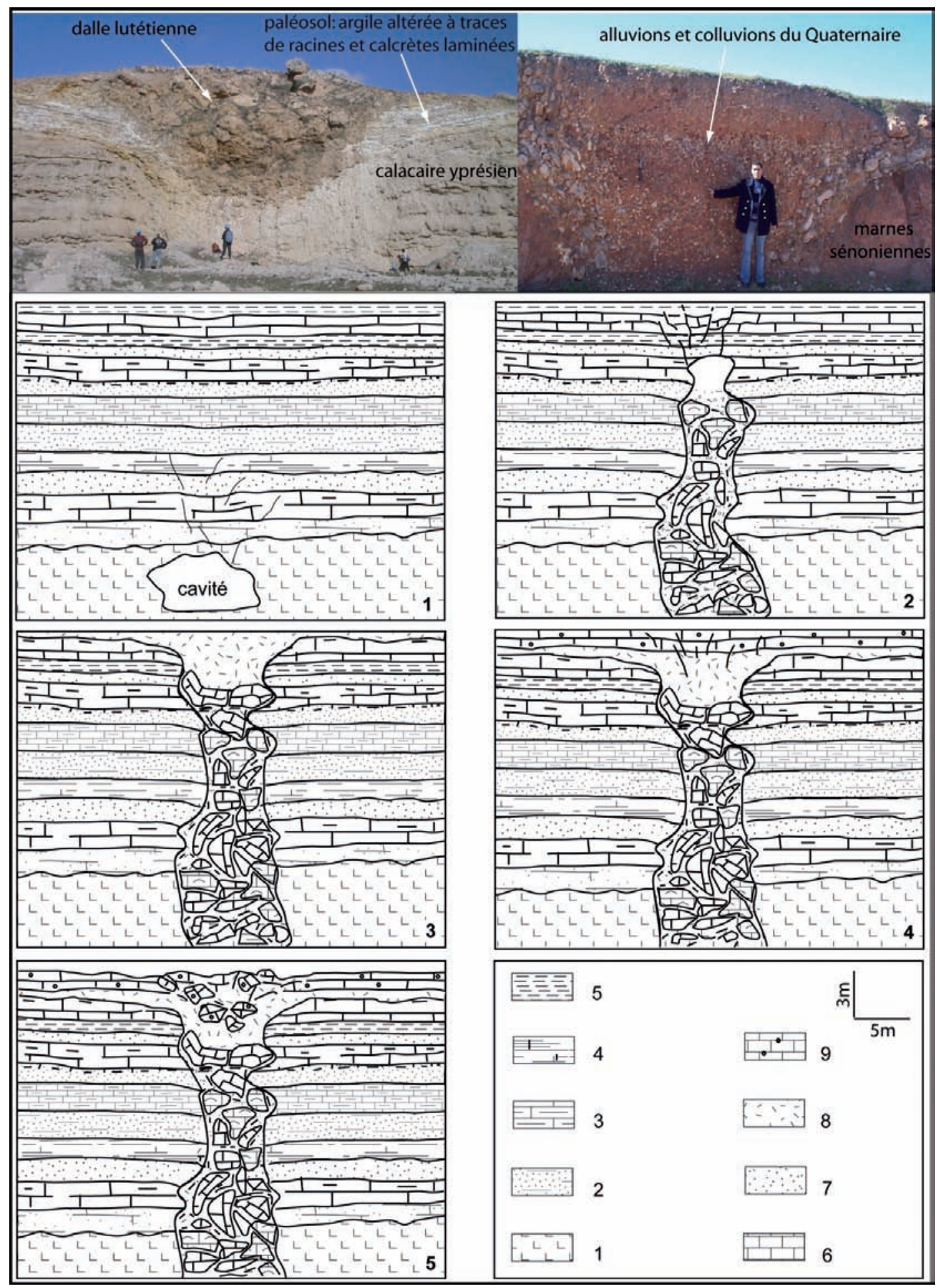

Fig. 9.-Exemple de paléosols observés au voisinage des zones dérangées dans les chantiers d'exploitation. Le karst yprisien est comblé par les calcaires lutétiens; de même le paléosol est compris entre ces deux âges. (Coupe Sidi Maati, localisation Fig. 1a). Le modèle proposé montre l'évolution chronologique du développement des karsts dans les formations évaporitiques sénoniennes qui constituent le soubassement de la série phosphatée dans le bassin des Ouled Abdoun depuis les stades précoces (apparition des cavités de dissolution dans le Sénonien) jusqu'aux stades de maturation de la formation du fontis. 1. Substrat gypseux 2 . Marnes phosphatées 3. Calcaire marneux 4. Marnes et silex 5. Marnes grises plastiques 6. Calcaires 7. Phosphates meubles 8. Remplissage pédogénétique du fontis 9. Calcaires à nodules de silex. 
tis, le profil d'altération pédologique montre parfois un épaississement considérable, pouvant aller jusqu'à 4 mètres d'épaisseur. Cette observation prouve que certains fontis ont été scellés par le (ou les) paléosol(s), même si des affaissements postérieurs ont fréquemment perturbé l'agencement initial de ces colmatages paléo-pédologiques.

En dehors des fontis eux même, il doit être noté que les faciès calcaires, dolomitiques ou phosphatés du Paléocène et de l'Yprésien ont diagénétiquement enregistré cette émersion, et cela jusqu'à une profondeur minimale d'une dizaine de mètres sous la surface de discontinuité. L'examen des lames minces sous cathodoluminescence montre qu'il existe des golfes de dissolution intercalés entre les phases de cimentation calcitiques désignées $\mathrm{Z} 1$ et Z2 (Fig. 6 e et f). La calcite Z1 est formée par de la sparite limpide et non luminescente qui est présente dans certaines phospharétites du Paléocène et dans les niveaux sus-jacents de l'Yprésien, mais pas dans la dalle lutétienne. La sparite limpide de la zone Z2, à luminescence orangée est, elle, présente dans tout l'intervalle Paléocène-Lutétien. En l'absence de mesures isotopiques ou de données micro-thermométriques sur les inclusions fluides, l'origine météorique ou marine de ces deux phases de cimentation ne peut être établie. En revanche, il est sûr que les golfes de corrosion qui affectent la calcite faiblement magnésienne de la zone $\mathrm{Z1}$ et scellés par la zone $\mathrm{Z} 2$ sont liés à une percolation de fluides météoriques sous saturés en carbonate de calcium.

Les observations macroscopiques et microscopiques, faites dans le cadre de ce travail, coroborent avec les résultats des travaux de Ambroggi (1963) et Piqué et al. (2007). Ces auteurs décrivent, dans plusieurs sites de la méseta marocaine (bassin des Ouled Abdoun, bassin des Gantours, bassin d'Essaouira), des faciès régressifs à la fin de l'Yprésien avant la dernière transgression à l'échelle du Maroc atlantique qui mettra en place la dalle calcaire à gastéropodes d'âge lutétien. La genèse du paléosol observé au toit de l'Yprésien à Sidi Maati peut raisonnablement être associée à cette régression. Les fluides météoriques agressifs ayant percolé à travers la série phosphatée, à la faveur de cette émersion, seraient à l'origine des figures de dissolutions et remplacements observées dans plusieurs lames examinées. Ces fluides ont vraisemblablement pu dissoudre les gypses du soubassement sénonien de la série phosphatée et donc engendrer la formation de fontis. Ces derniers ne sont autres que le résultat d'une réponse géomécamique des matériaux de cette série à la présence de vides sous jacents (Fig. 9).

\section{Karstifications post lutétiennes}

Les soutirages karstiques liés à la dissolution du gypse sénonien ne se sont de toute évidence pas arrêtés avec la mise en place de la dalle à Thersitées au Lutétien. Cette dalle est elle-même fréquemment brèchifiée et incorporée dans le remplissage des fontis (Fig. 5a \& 9), ce qui prouve que les carbonates lutétiens ont préalablement eu le temps d'être déposés puis cimentés. Dans certains fontis il apparaît que la dalle lutétienne a pu former une voûte mécaniquement stable au toit des fontis. Des "vides sous-lutétiens" sont alors apparus puis ont été partiellement ou totalement colmatés par des sédiments fins endokarstiques (Fig. 5a). Souvent silicifiés, ces sédiments sont nettement laminés mais perturbés par des soutirages karstiques synsédimentaires représentés par des petits slumps et par des petites failles listriques scellées par les lamines postérieures.

Des circulations météoriques à faveur d'une émersion post-lutétienne ont donc eu lieu et ont contributé au développement et la maturation des fontis observés dans les mines à ciel ouvert des phosphates. Les travaux de Piqué et al. (2007) montrent que la majeure partie des bassins atlantiques du Maroc est restée émergée depuis l'Eocène moyen. Par ailleurs, nous avons aussi observé entre Oued Zem et Sidi Daoui (localisation, Fig. 1a) des fontis développés dans le substrat gypseux du Sénonien et comblés par des formations superficielles quaternaires (colluvions, alluvions et limons de plateaux, Fig. 9). De telles observations prouvent que le phénomène de dissolution du gypse et collapse des formations sus-jacentes continu même actuellement.

\section{Bilan et Conclusions}

Cette étude a permis de préciser la nature et la chronologie des dérangements de la série phosphatée, et d'ébaucher un essai de l'évolution karstologique et diagénétique des gisements de phosphate dans le bassin sédimentaire des Ouled Abdoun. Elle montre pour la première fois que le Sénonien, qui constitue le soubassement de la série phosphatée dans ce bassin, est formé par une épaisse série évaportique à sub-évaporitique au sommet de laquelle 
ont été reconnues de nombreuses cavités karstiques, comblées ou non par les sédiments phosphatés susjacents. Les dérangements sont incontestablement des fontis qui se sont développés à la verticale de ces cavités souterraines. Les arguments géométriques relevés lors de cette étude et l'examen microscopique en cathodoluminescence montrent que l'histoire de formation de ces derniers est polyphasée:

Certaines cavités se sont formées dès le Sénonien, probablement à la faveur d'un épisode émersif à la fin de cet étage. Le développement d'une surface érosive, karstifiée, transpercée par des dépressions typiques d'un modelé épikarstique sur substrat évaporitique est bien observable dans la région d'Oued Zem. Cette surface dessine une discontinuité régionale entre les dépôts sénoniens gypseux et les phosphates maestrichtiens.

Les fontis affectant la série phosphatée sont formés à l'aplomb de cavités endokarstiques, développées dans le gypse sénonien à la faveur d'autres épisodes émersifs, probablement au toit de l'Yprésien ou encore après le dépôt de la dalle calcaire lutétienne. La circulation d'eau météorique à travers les diaclases des séries calcaréo-phosphatés a provoqué l'élargissement des cavités endokarstiques précitées et par suite l'effondrement de ces formations par processus gravitaires (collapses), ce qui a donné lieu aux dérangements rencontrés actuellement dans les chantiers d'exploitation. Dans le substrat évaporitique sénonien, on trouve aussi d'autres cavités encore plus récentes, remplies par des sédiments épikarstiques ou endokarstiques du Quartenaire. Les ébauches de modèles (Fig. 8 et 9) proposés pour expliquer la formation polyphasée des dérangements synthétisent les observations macroscopiques et microscopiques réalisées dans le cadre de cette étude et s'intégrent dans le contexte paléogéographique régional du Sénonien au Quaternaire. Un tel modèle sera encore affiné par les travaux en cours (thèse de mademoiselle N. El Assel).

La cartographie des dérangements de la série phosphatée, masqués par la couverture quaternaire, est une question de première priorité pour les ingénieurs miniers de l'OCP. Cette cartographie leur permettrait d'une part, de cerner les réserves de chaque gisement avant de démarrer son exploitation et de mieux orienter d'autre part, les tranchées d'exploitation pour contourner les zones les plus dérangées et gagner par conséquent au niveau du prix de revient de l'extraction des phosphates. Cette étude montre clairement que l'évolution et la répartition spatiale des dérangements dépendent très étroitement de l'épaisseur, de la position et de la distribution de la formation évaporitique sénonienne, à présent identifiée dans le bassin des Ouled Abdoun. Ainsi, il nous semble que la meilleure approche scientifique qui permettrait de résoudre le problème de cartographie des dérangements serait:

- A l'échelle du bassin des Ouled Abdoun: il est indispensable de mener une étude sédimentologique qui aboutira à l'élaboration d'une carte paléogéographique aussi précise que possible, avec cartes d'isopaques de la série des évaporites du Sénonien. Un tel travail devra intégrer toutes les données existantes en particulier celles des milliers de puits et forages traversant la série sénonienne disponibles dans le service géologique de l'OCP à Khoiuribga. La description lithologique exhaustive et étayée des niveaux sousjacents à la série phosphatée, qui reste très peu documentée, devra se faire en présence de spécialistes en évaporites et en karstologie. Cette étude est en cours de réalisation par mademoiselle Nora El Assel dans le cadre de ses travaux de doctorat.

- Une possible existence d'une structuration tectonique syn-sédimentaire, parallèle aux accidents majeurs du domaine mésetien marocain, est suspectée. Celle-ci aurait pu participer à la distribution des évaporites ainsi qu'à la formation d'un dense réseau de diaclases susceptibles d'influencer la distribution et la position des fontis. Il serait donc profitable d'engager une étude microstructurale couplée aux travaux sédimentologiques et paléogéographiques du Sénonien.

- A l'échelle de chaque gisement: en utilisant les données sédimentologiques et stratigraphiques, deux approches complémentaires pourraient permettre de prévoir, à l'échelle décamétrique, la position des dérangements dans la série phosphatée: (i) L'interprétation de photographies aériennes, outils fréquemment utilisée pour caractériser des fractures ou des systèmes de cavités comblées. En effet, sur le terrain, nous avons constaté qu'à l'aplomb des zones dérangées, le sol est souvent plus épais, humide et bien développé. On peut donc imaginer que la végétation qui y pousse sera en avance par rapport à celle en dehors. En conséquence, des photos aériennes prises à basse altitude (quelques centaines de mètres), effectuées au début de la croissance des végétaux, pourront permettre de délimiter les zones dérangées. (ii) Les études géophysiques expérimentales (résistivités, tomographie électrique et radar géologique) entreprises par l'équipe de l'Université de Marrakech (Maroc), de Pierre et Marie Curie-Paris VI (France) et de 
l'Institut Andaluz de Géophysique-Grenada (Espagne) (Kchikach et al., 2002 \& Kchikach et al., 2006) ont démontré la sensibilité du paramètre «résistivité électrique» pour tenter de cartographier et délimiter ces fontis sous couverture quaternaire. Etant donné qu'il est impossible de couvrir toute l'étendue des surfaces phosphatées reconnues (25000 ha, Fig. 1a), il serait judicieux d'orienter l'utilisation des méthodes géophysiques vers les zones potentiellement dérangées. Ces dernières ne sont autres que celles qui montrent une série évaporitique sénonienne épaisse et criblée de cavités de dissolution du gypse. Il faut alors développer et approfondir l'axe de recherche entreprise dans le présent travail en menant une étude sur la distribution, l'épaisseur et la position stratigraphique des gypses sénoniens. Cela permettrait de sélectionner à l'échelle des zones reconnues du secteur minier de Khouribga les endroits où la série phosphatée est susceptible d'être très dérangée (formations évaporitiques sénoniennes épaisses) de ceux où celle-ci serait plutôt régulière (formations évaporitiques sénoniennes réduites ou absentes).

\section{REMERCIEMENTS}

Les auteurs remercient vivement les reviewers de cet article pour leur conseils et remarques constructives qui ont contribué à l'amélioration significative du manuscrit. Cette étude a été réalisée grâce au soutien des Comités Mixtes Interuniversitaires Espagnol-Marocaine (Actions Intégrées $n^{\circ}$ A/025780/09 et $n^{\circ}$ A/031267/10) et Franco-Marocaine (Action Intégrée $n^{\circ}$ MA/09/209).

\section{Références}

Ambroggi, R. (1963): Etude géologique du versant méridional du Haut Atlas Occidental et de la plaine du Souss. Notes et Mémoires du Service Géologique du Maroc, 157, 321-338.

Amrhar, M. (1995). Tectonique et inversions géodynamiques post-rift dans le Haut Atlas occidental: structures, instabilités tectoniques et magmatisme liés à l'ouverture de l'Atlantique central et à la collision Afrique- Europe.structures, instabilités tectoniques et magmatisme liés à l'ouverture de l'Atlantique central et à la collision Afrique- Europe. PhD Thesis, Université Cadi Ayyad, Marrakech, Maroc, 252 pp.

Andreu, B. \& Tronchetti, G. (1994). Ostracodes et foraminifères du Crétacé supérieur du synclinal d'El Koubbat, Moyen Atlas, Maroc: Biostratigraphie, Paléoenvironements, Paléobiogéopgraphie, systématique des ostracodes. ler congrès français de stratigraphie CFS 94 Toulouse. Strata, 99-100.
Arenas, C.; Alonso-Zarza, A.M. \& Pardo, G. (1999). Dedolomitization and other early diagenetic processes in Miocene lacustrine deposits, Ebro Basin (Spain). Sedimentary Geology, 125: 23-45. doi:10.1016/S00370738(98)00146-8

Azmany, M. (1979). Évolution des faciès dans les gisements des Ouled Abdoun. Mines, Géologie et Energie, Rabat, 44: 35-38.

Azmany, M.; Farkhany, X. \& Salvan, H.M. (1986). Gisement des Ouled Abdoum, géologie des gites minéraux marocains. Notes et Mémoires du Service Géologique du Maroc, 276: 200-249.

Bardet, N.; Suberbiola, X.; Iarochene, M.; Bouyahyaoui, F.; Bouya, B. \& Amaghzaz, M. (2004). Mosasaurus beaugei from the Late Cretaceous phosphates of Morocco. Géobios, 37: 305- 401. doi:10.1016/j.geobios. 2003.02.006

Boujo, A. (2002). Abou shape and development of sterile bodies in phosphatic deposits. Comptes Rendus Géoscience, 334: 1113-1114. doi:10.1016/S1631-0713(02)01858-8

Boujo, A. (1976). Contribution à l'étude géologique du gisement de phosphate crétacé-éocène des Ganntour, Maroc occidentalnntour, Maroc occidental. PhD Thesis, Université Louis Pasteur de Strasbourg, Institut de géologie, $227 \mathrm{pp}$.

Braithwaite, C. J. R. \& Heath, R. A. (1992). Deposition and diagenesis of debris flows in Upper Ordovician limestones, Hadeland, Norway. Sedimentology, 39: 735767. doi:10.1111/j.1365-3091.1992.tb02151.x

Cazenave, S.; Chapoulie, R. \& Villeneuve, G. (2003). Cathodoluminescence of synthetic and natural calcite: the effects of manganese and iron on orange emission, Mineralogy and Petrology 78: 243-253. doi: 10.1007/s00710-002-0227-y

Cavin, L.; Bardet, N.; Cappetta, H.; Gheerbrant, E.; Iarochène, S.M. \& Sudre, J. (2000). A new Palaeocene albulid (Teleostei: Elopomorpha) from the Ouled Abdoun phosphatic basin, Morocco. Geological Magazine, 137: 583-591. doi:10.1017/S0016756800004647

Chapoulie, R.; Cazenave, S. \& Cerepi, A. (2005). Apport de la cathodoluminescence à haute résolution à l'étude de la diagenèse météorique dans les formations. Comptes Rendus Géoscience, 33: 337-346. doi:10.1016/j.crte.2004.10.008

Charroud, M. (1990). Evolution géodynamique de la partie sud-ouest du Moyen Atlas durant le Méso-Cénozoïque, Un exemple d'évolution intraplaque.ozoïque, Un exemple d'évolution intraplaque. PhD Thesis, Université Mohammed 5, Rabat, 232 pp.

Choubert, G. \& Salvan, H. (1949). Essai sur la paléogéographie du Sénonien au Maroc. Notes et Mémoires du Service Géologique du Maroc, 74: 13-50.

Choubert, G. \& Salvan, H.M. (1976). Evolution du Domaine atlasique marocain depuis les temps paléozoiques. Mémoires du Service Géologique de France, 1: 447-527.

Daoudi, L. (1996). Contrôle diagénétique et paléogéographique des argiles des sédiments mésozoïques $d u$ Maroc, comparaison avec les domaines atlantiques et 
téthysien du Maroc, comparaison avec les domaines atlantiques et téthysien. PhD Thesis, Université Cadi Ayyad, Marrakech, 250 pp.

Daoudi, L.; Deconink, J.F. (1994). Contrôles paléogéographique et diagénétique des successions sédimentaires argileuses du bassin atlasique au Crétacé (Haut Atlas occidental, Maroc). Journal of African Earth Sciences, 18: 123-134. doi:10.1016/0899-5362(94)90025-6

Daoudi, L.; Charroud, M.; Deconinck, J. F. \& Bouabde1li, M. (1995). Distribution et origine des minéraux argileux des formations crétacé-éocène du Moyen Atlas Sud-occidental (Maroc): Signification paléogéographique. Annales de la Societe Geologique du Nord, 4: 31-40.

De Putter, T.; Rouchy, J. M., Herbosch, A.; Keppens, E.; Pierre, C. \& Groessens, E. (1994). Sedimentology and palaeo-environment of the Upper Visean anhydrite formation of the Franco-Belgian. Carboniferous Basin (Saint-Ghislain borehole, southern Belgium). Sedimentary Geology, 90: 77-93. doi:10.1016/00370738(94)90018-3

Duffaud, F.; Brun, L. \& Fontbote, J.M. (1966). Le bassin du Sud-Ouest marocain. In: Bassins sédimentaires $d u$ litoral Africain (Reyre, D., ed.), Symposium New Delhi, Publications Associés du Service Géologique Africain, Paris, 1: 5-26.

Durlet, C.; Loreau, J. P. \& Pascel, A. (1992). Signature diagénétique des discontinuités et nouvelle représentation graphique de la diagenèse. Comptes Rendus Géoscience, 314: 1507-1514.

Dogan, U.; Özel, S. (2005). Gypsum karst and its evolution east of Hafik (Sivas, Turkey). Geomorphology, 71: 373-388. doi:10.1016/j.geomorph.2005.04.009

El Felah, E. (ed.) (1990). Étude des dérangements à Sidi Chennane. Rapport inédit, Service Méthodes et Planning, Division des Exploitations Minières de Khouribga, $25 \mathrm{pp}$.

El Hammichi, F.; Elmi, A. \& Faure-Muret, K. (2002). Une plate-forme en distension, témoin de phases pré-accrétion téthysienne en Afrique du nord pendant le Toarcien-Aalenien (synclinal Iguer Awragh-Afennourir, Moyen Atlas occidental, Maroc). Comptes Rendus Géoscience, 334: 1003-1010. doi:10.1016/S16310713(02)01841-2

El Mountassir, M. (1977). La zone rubéfiée de Sidi Daoui, altération météorique du phosphate des Oulad Abdoun (Maroc). PhD Thesis, Université Louis-Pasteur, Strasbourg, 126 pp.

Elorza, J. J. \& Rodriguez-Lazaro, J. (1984). Late Cretaceous quartz geodes after anhydrite from Burgos, Spain. Geological Magazine, 121: 107-113. doi:10.1017/S0016756800028077

Elorza, M.G. \& Santolalla F.G. (1998). Geomorphology of the Tertiary gypsum formations in the Ebro depression (Spain), Geoderma, 87: 11-29. doi:10.1016/S00167061(98)00065-2

Emery, D. \& Dickson, J.-A. (1989). A syndepositional meteoric phreatic lens in the Middle Jurassic Lincolnshire Limestone, England, UK, Sedimentary Geol. 65: 273-274. doi:10.1016/0037-0738(89)90029-8
Ettazarini, S. (2004). Incidences of water-rock interaction on natural resources characters, Oum Er-Rabia Basin (Morocco). Environmental Geology, 47: 69-75. doi:10.1007/s00254-004-1129-6

Fedan, B. (1989). Evolution géodynamique d'un bassin intraplaque sur décrochements: le Moyen Atlas durant le Méso-Cénozoique. Phd Thesis, Université Mohammed V, Rabat, 338 pp.

Gheerbrant, E.; Sudre, J.; Cappetta, H.; Mourer-Chauviré, C.; Bourdon, E.; Iarochene, M., Amaghzaz, M. \& Bouya, B. (2003). Les localités à mammifères des carrières du Grand Daoui, bassin des Ouled Abdoum, Maroc. Bulletin de la Société Géologique de France, 174: 279-294. doi:10.2113/174.3.279

Hardenbol, J.; Thierry, J.; Farley, M.B.; Jacquin, T.; Graciansky, P.C. \& Vail, P.R. (1998). Mesozoic and Cenozoic sequence chronostratigraphic framework of european basins. In: Mesozoic and Cenozoic Sequence Stratigraphy of European Basins. (Graciansky, P.C.; Hardenbol, J.; Jacquin, T. \& Vail, P.R., Eds.), SEPM Special Publication 60, Charts 2-8. doi: 10.2110/pec.98.60.

Huvelin, P. (1973). Mouvements pré-atlasiques, atlasiques et récents dans les Jbilet et sur leur poutours. Notes $d u$ Service Géologique du Maroc, 33: 83-123.

Hsissou, Y.; Chauve, P. \& Mania, J. (1996). The aquifer of Turonian limestones (Tadla Basin, Morocco). Local and remote groundwater recharge from the Atlas. Journal of Hydrology, 183: 433-443. doi:10.1016/00221694(95)03010-7

Kchikach, A.; Jaffal, M.; Aïf, T. \& Bahi, L. (2002). Cartographie de corps stériles sous couverture quaternaire par méthode de résistivités électriques dans le gisement phosphaté de Sidi Chennane, Maroc. Comptes Rendus Géoscience, 334: 379-386. doi:10.1016/S16310713(02)01767-4

Kchikach, A.; Andrieux, P.; Jaffal, M.; Amrhar, M.; Mchichi, M.; Bouya, B.; Amaghzaz, M.; Veyrieras, T. \& Iqizou, K. (2006). Les sondages électromagnétiques temporels comme outil de reconnaissance du gisement phosphaté de Sidi Chennane (Maroc): Apport à la résolution d'un problème d'exploitation. Comptes Rendus Géoscience, 338: 289-296. doi:10.1016/j.crte.2006.02.003

Klimchouk, A. (1996). The typology of gypsum karst according to its geological and geomorphological evolution. Int. J. Speleol., 25: 49-59.

Laville, E. (1985). Evolution sédimentaire, tectonique et magmatique du bassin jurassique du Haut-Atlas, Maroc: Modèle en relais multiples de décrochements. $\mathrm{PhD}$ Thesis, Montpellier, $168 \mathrm{pp}$.

Laville, E. \& Piqué, A. (1992). Jurassic penetrative deformation and Cenozoic uplift in the Central High Atlas (Morocco): a tectonic model. Structural and Orogenic inversions: Geologische Rundschau, 81: 157-170. doi:10.1007/BF01764546

Le Roy, P. (1997). Les bassins ouest marocains; leur formation et leur évolution dans le cadre de l'ouverture et du développement de l'Atlantique central (marge afri- 
caine). PhD Thesis, Univ. de Bretagne Occidentale, Brest, 327 pp.

Lucas, J.; Prévôt, L. \& El Mountassir, M. (1979). Les phosphorites rubéfiées de Sidi Daoui. Transformation météorique locale du gisement de phosphate des Oulad Abdoun (Maroc). Bulletin Sciences Géologiques,32: 21-37.

Machel, H. G. (1985). Cathodoluminescence in calcite and dolomite and its chemical interpretation. Géoscience Canada, 12: 139-147.

Michard, A. (1976). Eléments de géologie marocaine. Notes et Mémoires du Service Géologique du Maroc, 252: 217-245.

Mazzulo, S. L.; Bischoff, W. D. \& Lobitez, H. (1990). Diagenesis of radiaxial fibrous calcites in a subconformity, shallow-burial setting: Upper Triassic an Liassic, Northern Calcareous Alps, Austria. Sedimentology, 37: 407-425. doi:10.1111/j.1365-3091.1990.tb00144.x

Moutaouakil, D. (1990). Sédimentologie et minéralogie des phosphates céno-mésozö̈ques du sud du bassin des Ouled Abdoun (Maroc): Application à la géochimie de l'Uranium. PhD Thesis, Université Cadi Ayyad, 340 pp.

Moutaouakil, D. \& Giresse, P. (1993). Petrology and sedimentary environment of mesocenozoic phosphorites of Ouled Abdoun. Bulletin de la Société Géologique de France, 164: 473-491.

Noubhani, A. \& Cappetta, H. (1995). Les Orectolobiformes, Carcharhiniformes et Myliobatiformes (Elasmobranchii, Neoselachii) des bassins à phosphate du Maroc (Maastrichtien-Lutétien basal). Palaeo Ichthyologica, 8: 315-327.

Orozco, M.; Molina, J. M. \& Crespo-Blanc, A. (1999). Palaeokarst and rauhwacke development, mountain uplift and subaerial sliding of tectonic sheets (northern Sierra de los Filabres, Betic Cordilleras, Spain), Springer Science, 78: 103-117.

Piqué, A.; Soulaimani, A.; Hoepffner, C.; Bouabdelli, M.; Laville, E.; Amrhar M. \& Chalouan, A. (ed.) (2007). Géologie du Maroc (nouvelle édition). Géode Editions, Marrakech, 287 pp.

Piqué, A.; Ait Brahim, L.; Ait Ouali, R.; Amrhar, M.; Charroud, M.; Gourmelen, C.; Laville, E.; Rekhiss, F. \& Tricart, P. (1998). Evolution structurale des domaines atlasiques du Maghreb au Méso-Cénozoïque; le rôle des structures héritées dans la déformation du domaine atlasique de l'Afrique du Nord. Bulletin de la Societé Géolique de France, 6: 797-810.

Rodriguez-Aranda, J.P.; Calvo, J.P. \& Esther Sanz-Montero, M. (2002). Paleosubsidence and active subsidence due to evaporite dissolution in the Zaragoza area (Huerva River valley, NE Spain): processes, spatial distribution and protection measures for transport routes. Sedimentology, 49: 1385-1400. doi:10.1046/j.13653091.2002.00504.x

Salvan, H.M. (ed.) (1963). Étude complémentaire sur le gisement de Merah El Areh. Etude d'ensemble des niveaux supérieurs. Rapport inédit, Document interne OCP, 14 pp.

Salvan, H. (1982). Phosphates. In: Géologie des gites minéraux marocains. Notes et Memoires du Service Géolique du Maroc, 87: 283-320.

Salvan, H., Farkhany, M., (1982) Les phosphates de chaux sédimentaires du maroc. Leurs caractéristiques et leurs problèmes (essai de synthèse). Notes et Mémoires $d u$ Service Géologique du Maroc, 14: 7-20.

Salvany, J. M.; Munoz, A. \& Perez, A. (1994). Nonmarine evaporitic sedimentation and associated diagenetic processes of the southwestern margin of margin of Ebro Basin (Lower Miocene), Spain. Journal of Sedimentary Petrology, 64: 190-203.

Sheldon, A.J.G. \& Davidson, R.P. (1989). The phosphates basins of Morocco. In: Phosphate deposits of the world. (Notholt, A.J.G., Ed.) Cambridge University Press, Cambridge, 2: 301-311.

Suberbiola-Pereda, X.; Bardet, N.; Jouve, S.; Iarochène, M.; Bouya, B. \& Amaghzaz, M. (2003). A new azhdarchid pterosaur from the Late Cretaceous phosphates of Morocco. Journal of the Geological Society of London, Special Publication, 217: 79-90. doi:10.1144/GSL.SP.2003.217.01.08

Wiedmann, J.; Butt, A.; \& Einsele, G. (1982). Cretaceous stratigraphy, environment and subsidence history of the morrocan continental margin. In: Geology of the Northwest Africa continental margin. (Samthein, M. \& Seibold, E., eds.). Springer verlag, Berlin, 366-395. doi:10.1007/978-3-642-68409-8_15

Zouhri, S.; Kchikach, A.; Saddiqi, O.; El Haımer, F.Z.; Baidder, L. \& Michard, A. (2008). The Cretaceous-tertiary plateaus. In: Continental Evolution-The Geology of Morocco: Structure, Stratigraphy, and Tectonics of the Africa-Atlantic-Mediterranean. (Michard, A.; Saddiqi, O.; Chalouan, A.; Frizon de Lamotte, D., Eds., Springer verlag, eds.), 331-358.

Recibido el 26 de agosto de 2011 Aceptado el 28 de febrero de 2012 Publicado online el 26 de junio de 2012 
